\title{
Multistability of a Two-Dimensional Map Arising in an Influenza Model
}

\author{
Yu-Jhe Huang ${ }^{1} \cdot$ Hsuan Te Huang $^{1} \cdot$ Jonq Juang $^{1}\left(\mathbb{D} \cdot\right.$ Cheng-Han Wu$^{1}$
}

Received: 7 June 2021 / Accepted: 17 December 2021 / Published online: 28 December 2021

(c) The Author(s), under exclusive licence to Springer Science+Business Media, LLC, part of Springer Nature 2021

\begin{abstract}
In this paper, we propose and analyze a nonsmoothly two-dimensional map arising in a seasonal influenza model. Such map consists of both linear and nonlinear dynamics depending on where the map acts on its domain. The map exhibits a complicated and unpredictable dynamics such as fixed points, period points, chaotic attractors, or multistability depending on the ranges of a certain parameters. Surprisingly, bistable states include not only the coexistence of a stable fixed point and stable period three points but also that of stable period three points and a chaotic attractor. Among other things, we are able to prove rigorously the coexistence of the stable equilibrium and stable period three points for a certain range of the parameters. Our results also indicate that heterogeneity of the population drives the complication and unpredictability of the dynamics. Specifically, the most complex dynamics occur when the underlying basic reproduction number with respect to our model is an intermediate value and the large portion of the population in the same compartment changes in states the following season.
\end{abstract}

Keywords Seasonal influenza model $\cdot$ Nonsmoothly two-dimensional map $\cdot$ Periodic points $\cdot$ Chaotic attractors $\cdot$ Multistability

Mathematics Subject Classification 37N25 - 92B

$凶 \quad$ Jonq Juang

jjuang@math.nctu.edu.tw

Yu-Jhe Huang

yjhuang@math.nctu.edu.tw

Hsuan Te Huang

gary91046@gmail.com

Cheng-Han Wu

u247921346@gmail.com

1 Department of Applied Mathematics, National Yang Ming Chiao Tung University, Hsinchu 300, Taiwan, ROC 


\section{Introduction}

The theory of dynamical systems is a major mathematical discipline closely intertwined with most of the main areas of mathematics. Furthermore, its concepts and methods can be applied to a wide variety of topics such as epidemiology, biology, medicine, physics, chemistry, finance, and more (Strogatz 2001; Jackson and Radunskaya 2015; Agarwal 1995; Swishchuk and Islam 2013). The investigation of the dynamical systems has also revealed an interesting phenomenon, namely multistability behaviors or coexisting attractors (Natiq et al. 2018, 2019; Rahim et al. 2019; Martins and Gallas 2008; Casas and Rech 2012; Zhang and Luo 2018; Bao et al. 2021; Li et al. 2020a; Gilardi-Velázquez et al. 2017; Anzo-Hernández et al. 2018; Escalante-González and Campos 2020). Multistability is the characteristic of a system presenting two or more mutually exclusive stable states. Bistable systems, for example, enable the implementation of logic gates (Santos-Moreno et al. 2020) and therefore computation. The applications of multistability can also be found in the field of image processing (Morfu et al. 2007) and morphing aircraft (Weisshaar 2013; Mattioni et al. 2008).

In this paper, we investigate a two-dimensional map that consists of both linear and nonlinear dynamics depending on where the map acts on its domain. It can be used to describe the dynamics of the season-to-season epidemic for an influenza. The derivation of the map is given in appendix. The two-dimensional map is also capable of generating very rich dynamics. For instance, such map displays a variety of qualitatively different dynamics: fixed points, period solutions, chaotic attractors, or bistable states. Surprisingly, bistable states include not only the coexistence of a stable fixed point and stable period three points but also that of stable period three points and a chaotic attractor. The goal of the paper is to classify the effect of the parameters on the dynamics of the model and to provide their theoretical analysis.

Our theoretical main results contain the following. First, the existence, uniqueness, and stability of the fixed point are established. Moreover, we also show that if the adjusted basic reproduction $\bar{r}_{0}$ is small, say $\bar{r}_{0} \leq 1$, then the disease dies out. On the other hand, if $\bar{r}_{0}$ is sufficiently large, then the epidemic returns every year. Second, the existence and nonexistence of a special type of period $n$ points, epidemic returns once every $n$ years, are obtained. Note that such special type, denoted by $3(0,0,+)$ type, of stable period three points may coexist with the nontrivial stable fixed point as well as with a chaotic attractor at certain range of parameters, respectively. We shall term, respectively, the above-mentioned phenomena as $(1,3(0,0,+))$ - and $(3(0,0,+), \mathrm{C})$ stability. Third, we prove the existence of $(1,3(0,0,+))$-stability over a wide range of parameters and also provide the possible range of parameters for producing the $(3(0,0,+), C)$-stability. It should be remarked that the verification of chaotic attractors is done by computing their Lyapunov exponents numerically. In summary, our theoretical and numerical results seem to suggest that great heterogeneity of the population contributes to the occurrence of the complication and unpredictability of the dynamics. Otherwise, the dynamics is much less complicated.

We conclude the introductory section by mentioning the organization of the paper. Our main results are contained in Sect. 2. We also provide the bifurcation diagram of one of the state variables versus $\bar{r}_{0}$. Some interesting and complicated dynamics could 
be identified including but not limited to, other type of multistability, at the end of

Sect. 2. The derivation of the model map is placed on the appendix. Some concluding remarks concerning the physical discussions and interpretations of the dynamics of the model as well as some related open problems are stated in Sect. 3.

\section{Dynamics of the Two-Dimensional Map}

Of concern is a discrete iterative map defined in the following.

$$
\left[\begin{array}{l}
x_{n+1} \\
y_{n+1}
\end{array}\right]=\mathbf{C}\left[\begin{array}{l}
x_{n} \\
y_{n}
\end{array}\right]+\left[\begin{array}{l}
c_{2} p_{n} \\
c_{3} p_{n}
\end{array}\right]:=\mathbf{F}\left[\begin{array}{l}
x_{n} \\
y_{n}
\end{array}\right]
$$

where

$$
\mathbf{C}=\left[\begin{array}{cc}
c_{0} & 0 \\
1-c_{0} & c_{1}
\end{array}\right]
$$

and

$$
p_{n}= \begin{cases}\frac{2}{\bar{r}_{0}}\left(1-\frac{1}{\bar{r}_{0}\left(1-x_{n}-y_{n}\right)}\right), & \text { if } x_{n}+y_{n} \leq 1-\frac{1}{\bar{r}_{0}}, x_{n}, y_{n} \geq 0 \\ 0, & \text { if } 1-\frac{1}{\bar{r}_{0}} \leq x_{n}+y_{n} \leq 1, x_{n}, y_{n} \geq 0\end{cases}
$$

Here $p_{n}, x_{n}$, and $y_{n}$ are, respectively, the proportion of the infected population, the proportion of the quickly and slowly recovered populations at time $n$. For $p_{n}=0$ (resp., $>0$ ), it is said that the epidemic dies out (resp., occurs) at time $n . \bar{r}_{0}$, a positive number, denotes the adjusted basic reproduction numbers. If $0<\bar{r}_{0} \leq 1$, then we see, via (1c), $p_{n}=0$ for all $n$, meaning that the epidemic will never occur. The parameters $c_{i}, 0 \leq c_{i}<1, i=0,1,2,3$, and $c_{2}+c_{3} \leq 1$, gives the measurement of the movements from $p_{n}, x_{n}$ and $y_{n}$ to $x_{n+1}$ and $y_{n+1}$. The detailed derivation of the equation in (1c) can be found in appendix. The dynamics of model $(1 \mathrm{a}, 1 \mathrm{~b})$ is to be investigated in this section. We begin with deriving conditions that $T:=\{(x, y): x, y \geq 0$ and $x+y \leq 1\}$ is invariant under the map $\mathbf{F}$ defined in (1a).

Proposition 2.1 Let $0 \leq c_{0}, c_{1}<1, c_{2}, c_{3} \geq 0$ and $c_{2}+c_{3} \leq 1$. The region $T$ is invariant under the map $\boldsymbol{F}$.

Proof We first consider the case that $\bar{r}_{0} \geq 1$. Suppose $\left(x_{0}, y_{0}\right) \in T$. Then,

$$
x_{1}+y_{1}=x_{0}+c_{1} y_{0}+\frac{2\left(c_{2}+c_{3}\right)}{\bar{r}_{0}}-\frac{2\left(c_{2}+c_{3}\right)}{\bar{r}_{0}^{2}\left(1-x_{0}-y_{0}\right)} \text {. }
$$

Let

$$
f_{1}(x)=x+a-\frac{b}{1-x}
$$


where $a=\frac{2\left(c_{2}+c_{3}\right)}{\bar{r}_{0}}$ and $b=\frac{2\left(c_{2}+c_{3}\right)}{\bar{r}_{0}^{2}}$. Then, $f_{1}(x), 0<x<1$, has a maximum at $1-\sqrt{b}$ or 0 depending on whether $b \leq 1$ or $b>1$. Clearly, if $b \leq 1$, then $f_{1}(1-\sqrt{b})=1-2 \sqrt{b}+a \leq 1$. Now, if $b>1$, then

$$
f_{1}(0)=a-b=2\left(c_{2}+c_{3}\right)\left(\frac{1}{\bar{r}_{0}}-\frac{1}{\bar{r}_{0}^{2}}\right) \leq \frac{1}{2}\left(c_{2}+c_{3}\right) \leq 1 .
$$

Hence, $x_{1}+y_{1} \leq 1$. Obviously, $x_{1}, y_{1} \geq 0$. For $\bar{r}_{0} \leq 1$, then the map $\mathbf{F}$ reduces to the linear map $\mathbf{C}$. The assertion of the proposition can then easily verified. The proof of the proposition is now completed.

We next address the question of the existence for the fixed point of the map $\mathbf{F}$ in the region $\mathrm{S}$. To simplify the calculation, we shall, from here on, assume that $c_{0}=c_{1}$. It should also be noted that many interesting and complicated dynamics can still be captured with such simplified assumption. For $\bar{r}_{0}>1$, let

$$
T_{\bar{r}_{0}}=\left\{(x, y): x+y \leq 1-\frac{1}{\bar{r}_{0}}, x, y \geq 0\right\} .
$$

Proposition 2.2 The map $\boldsymbol{F}$ defined in (1a) exists only one fixed point in T. In particular, if $\bar{r}_{0}>1$, then such fixed point exists in $T_{\bar{r}_{0}}$.

Proof If $\bar{r}_{0} \leq 1$, then the map $\mathbf{F}$ reduces to the linear map $\mathbf{C}$, which has a unique fixed point in the origin. We next consider the case that $\bar{r}_{0}>1$. For such case, the only possible candidate for the fixed point of $\mathbf{F}$ lies in $T_{\bar{r}_{0}}$. Let $(x, y, p)=\left(x_{*}, y_{*}, p_{*}\right)$, where $\left(x_{*}, y_{*}\right)$ is a fixed point of the map $\mathbf{F}$. Then, $x_{*}=\frac{c_{2}}{1-c_{1}} p_{*}$ and $y_{*}=\frac{c_{2}+c_{3}}{1-c_{1}} p_{*}$. Let

$$
c=\frac{2 c_{2}+c_{3}}{1-c_{1}}
$$

Upon using (1c), we have that $p_{*}$ satisfies the equation $1=\left(1-c p_{*}\right)\left(\bar{r}_{0}-\frac{\bar{r}_{0}^{2}}{2} p_{*}\right)$. Solving the above equation, we get, for $c \neq 0$,

$$
p_{*}=\frac{1}{2 c \bar{r}_{0}}\left[2 c+\bar{r}_{0} \pm \sqrt{8 c+\left(2 c-\bar{r}_{0}\right)^{2}}\right]=: p_{ \pm} .
$$

Clearly, $x_{*}+y_{*}\left(=c p_{*}\right)$ should be less than one for any feasible fixed point $\left(x_{*}, y_{*}\right) \in$ $T_{\bar{r}_{0}}$. However, $c p_{+}>1$ for any $c \in[0, \infty)$. To see this, we compute $\frac{\partial\left(c p_{+}\right)}{\partial c}$. The resulting calculation yields that $c p_{+}$is strictly increasing in $c$. Since $\left.c p_{+}\right|_{c=0}=1$, we have that $c p_{+}>1$. Thus, $\left(x_{+}, y_{+}\right)$is not a feasible fixed point for the map defined in (1), where $x_{+}=\frac{c_{2}}{1-c_{1}} p_{+}$and $y_{+}=\frac{c_{2}+c_{3}}{1-c_{1}} p_{+}$. Likewise, we define $x_{-}$and $y_{-}$ similarly. To complete the proof, it remains to show that $\left(x_{-}, y_{-}\right)$is a feasible fixed point. Some direct calculation would yield that $c p_{-}<1-\frac{1}{\bar{r}_{0}}$. Now, if $c \geq \frac{\bar{r}_{0}}{2\left(\bar{r}_{0}-1\right)}$ 
or equivalently $2 c \bar{r}_{0} \geq 2 c+\bar{r}_{0}$, then $p_{-}<1$ as desired. If $0 \leq c<\frac{\bar{r}_{0}}{2\left(\bar{r}_{0}-1\right)}$, then $p_{-}<1$ provided that

$$
(1-c)\left(\bar{r}_{0}^{2}-2 \bar{r}_{0}\right)+2>0 .
$$

The inequality above is clearly satisfied whenever $0 \leq c \leq 1$ for any $\bar{r}_{0}>1$. For $c>1$, we must have that $\bar{r}_{0} \leq 2$. Otherwise, $c<\frac{\bar{r}_{0}}{2\left(\bar{r}_{0}-1\right)} \leq 1$, a contradiction. However, if $1<\bar{r}_{0} \leq 2$ and $c>1$, then the inequality in (4) holds true as well. We just completed the proof of the proposition.

Remark 2.1 (a) The fixed point mentioned in Proposition 2.2 has the following form $x_{*}=\frac{c_{2}}{1-c_{1}} p_{-}$, and $y_{*}=\frac{c_{2}+c_{3}}{1-c_{1}} p_{-}$, where $p_{-}$is defined in $(3 \mathrm{~b})$.

(b) For $\bar{r}_{0} \leq 1$, the map $\mathbf{F}$ reduces to the linear map $\mathbf{C}$. Consequently, the epidemic dies out each season.

We next seek to find the conditions for the existence of a special form of period $n$ point. Specifically, a period $n$ solution when there is an epidemic once in every $n$ years is to be sought, i.e., the infected population sizes are all zero at all $n$ seasons except one. For necessity, we set $x_{k, m, n}$ and $y_{k, m, n}$ to be the size of $R^{\phi}(=x)$ and $R^{1}(=y)$, respectively, at the $k$-th season with the nonzero infected population size occurring at the $m$-th season, where $0 \leq k, m \leq n-1$. We further assume that its infected population size $p_{m}$ at the $m$-th season is equal to $\bar{p}_{m, n}$. Should no ambiguity arise, we shall write $\bar{p}_{m, n}=\bar{p}_{n}, x_{k, m, n}=x_{k, m}$ and $y_{k, m, n}=y_{k, m}$. Then,

$$
\left(\begin{array}{l}
x_{0, m} \\
y_{0, m}
\end{array}\right)=\mathbf{C}^{n}\left(\begin{array}{l}
x_{0, m} \\
y_{0, m}
\end{array}\right)+\bar{p}_{n} \mathbf{C}^{n-m-1}\left(\begin{array}{l}
c_{2} \\
c_{3}
\end{array}\right),
$$

and so

$$
\left(\begin{array}{l}
x_{0, m} \\
y_{0, m}
\end{array}\right)=\bar{p}_{n}\left(\mathbf{I}-\mathbf{C}^{n}\right)^{-1} \mathbf{C}^{n-m-1}\left(\begin{array}{l}
c_{2} \\
c_{3}
\end{array}\right)
$$

Now,

$$
\begin{aligned}
\left(\mathbf{I}-\mathbf{C}^{n}\right)^{-1} \mathbf{C}^{n-m-1} & =\sum_{k=1}^{\infty} \mathbf{C}^{k n-m-1} \\
& =\sum_{k=1}^{\infty}\left[c_{1} \mathbf{I}+\left(\begin{array}{cr}
0 & 0 \\
1-c_{1} & 0
\end{array}\right)\right]^{k n-m-1} \\
& =\frac{c_{1}^{n-m-1}}{1-c_{1}^{n} \mathbf{I}+s_{n, m}\left(\begin{array}{cc}
0 & 0 \\
1-c_{1} & 0
\end{array}\right)} \\
& =\left(\begin{array}{cc}
\frac{c_{1}^{n-m-1}}{1-c_{1}^{n}} & 0 \\
\left(1-c_{1}\right) s_{n, m} & \frac{c_{1}^{n-m-1}}{1-c_{1}^{n}}
\end{array}\right)
\end{aligned}
$$


Here

$$
\begin{aligned}
s_{n, m} & =\sum_{k=1}^{\infty}(k n-m-1) c_{1}^{k n-m-2} \\
& =\sum_{k=1}^{\infty}(n-m-1) c_{1}^{k n-m-2}+\sum_{k=1}^{\infty}(k-1) n c_{1}^{k n-m-2} \\
& =\frac{(n-m-1) c_{1}^{n-m-2}}{1-c_{1}^{n}}+\frac{n c_{1}^{2 n-m-2}}{\left(1-c_{1}^{n}\right)^{2}} \\
& =\frac{1}{\left(1-c_{1}^{n}\right)^{2}}\left[(n-m-1) c_{1}^{n-m-2}+(m+1) c_{1}^{2 n-m-2}\right] .
\end{aligned}
$$

Consequently, it follows from (5a) that

$$
\begin{aligned}
& x_{0, m}=\frac{c_{2} c_{1}^{n-m-1}}{1-c_{1}^{n}} \bar{p}_{n} \text { and } \\
& y_{0, m}=\left(s_{n, m} c_{2}\left(1-c_{1}\right)+\frac{c_{3} c_{1}^{n-m-1}}{1-c_{1}^{n}}\right) \bar{p}_{n} .
\end{aligned}
$$

Let

$$
\begin{aligned}
t_{n, m}: & =\left\|\left(\mathbf{I}-\mathbf{C}^{n}\right)^{-1} \mathbf{C}^{n-m-1}\left(\begin{array}{l}
c_{2} \\
c_{3}
\end{array}\right)\right\|_{1} \\
& =\frac{1}{\bar{p}_{n}}\left(x_{0, m}+y_{0, m}\right) \\
& =\frac{\left(c_{2}+c_{3}\right) c_{1}^{n-m-1}}{1-c_{1}^{n}}+s_{n, m} c_{2}\left(1-c_{1}\right) .
\end{aligned}
$$

Set $m=0$. Then, $\bar{p}_{n}$ satisfies the following equation

$$
\bar{p}_{n}=\frac{2}{\bar{r}_{0}}\left(1-\frac{1}{\bar{r}_{0}\left(1-\bar{p}_{n} t_{n, 0}\right)}\right) \text {. }
$$

Consequently,

$$
\bar{p}_{n}=\frac{\left(1+\frac{2}{\bar{r}_{0}} t_{n, 0}\right)-\sqrt{\left(1-\frac{2}{\bar{r}_{0}} t_{n, 0}\right)^{2}+\frac{8}{\bar{r}_{0}^{2}} t_{n, 0}}}{2 t_{n, 0}}
$$

Note that another root of equation (5e) is not feasible. Using (5a), we have that

$$
x_{i, 0}=x_{0, n-i} \text { and } y_{i, 0}=y_{0, n-i} i=1,2, \ldots n-1 \text {. }
$$


Now, to verify the existence of the period $n$ solution with $p_{0}=\bar{p}_{n}, p_{1}=p_{2}=$ $\cdots p_{n-1}=0$, it remains to show that

$$
x_{0,0}+y_{0,0} \leq 1-\frac{1}{\bar{r}_{0}},
$$

and

$$
\begin{aligned}
x_{i, 0}+y_{i, 0} & =x_{0, n-i}+y_{0, n-i} \\
& =\bar{p}_{n} t_{n, n-i} \geq 1-\frac{1}{\bar{r}_{0}} . \quad i=1,2, \ldots n-1 .
\end{aligned}
$$

We have used (6b) to justify the first equality in (6d). The following proposition is to show that for fixed $n, t_{n, n-i}$ is decreasing in $i$. Hence, if (6d) is satisfied for $i=n-1$, then (6d) holds true for all $i=1,2, \ldots, n-1$.

Proposition 2.3 For fixed $n$ and $0<c_{1}<1$, the inequality $t_{n, m}>t_{n, m-1}$ holds true for $m=1,2, \ldots, n-1$.

Proof Using (5d), we have that

$$
\begin{aligned}
& t_{n, m}-t_{n, m-1}=\frac{\left(c_{2}+c_{3}\right) c_{1}^{n-m-1}\left(1-c_{1}\right)}{1-c_{1}^{n}}+ \\
& \frac{c_{1}^{n-m-2} c_{2}\left(1-c_{1}\right)}{\left(1-c_{1}^{n}\right)^{2}} \times\left[(n-m-1)+(m+1) c_{1}^{n}-m c_{1}^{n+1}-(n-m) c_{1}\right] .
\end{aligned}
$$

The above term is positive provided that $c_{1}\left(1-c_{1}^{n}\right)+n-m-1+(m+1) c_{1}^{n}-m c_{1}^{n+1}-$ $(n-m) c_{1}>0$. Rearranging the right hand side of the above inequality, we get that $(n-m)\left(1-c_{1}\right)+m c_{1}^{n}\left(1-c_{1}\right)-\left(1-c_{1}^{n}\right)\left(1-c_{1}\right)=\left(1-c_{1}\right)\left(n-m+m c_{1}^{n}-1+c_{1}^{n}\right)$, which is, indeed, greater than zero whenever $0<c_{1}<1$. The proof of the proposition is complete.

Clearly, (6c) is equivalent to

$$
\left(1+\frac{2}{\bar{r}_{0}} t_{n, 0}\right)-\left(\left(1-\frac{2}{\bar{r}_{0}} t_{n, 0}\right)^{2}+\frac{8}{\bar{r}_{0}^{2}} t_{n, 0}\right)^{\frac{1}{2}} \leq 2\left(1-\frac{1}{\bar{r}_{0}}\right) .
$$

Some direct calculation from the above inequality would yield that (6c) is always satisfied for any $n$ as long as $\bar{r}_{0} \geq 1$. For $i=n-1$, we have, via Proposition 2.3, (5c) and $(5 \mathrm{~d})$ that $(6 \mathrm{~d})$ becomes

$$
\begin{aligned}
t_{n, 1}\left(1+\frac{2}{\bar{r}_{0}} t_{n, 0}\right) & -2 t_{n, 0}\left(1-\frac{1}{\bar{r}_{0}}\right) \\
& \geq t_{n, 1}\left(\left(1-\frac{2}{\bar{r}_{0}} t_{n, 0}\right)^{2}+\frac{8}{\bar{r}_{0}^{2}} t_{n, 0}\right)^{\frac{1}{2}} .
\end{aligned}
$$


For (7a) to be satisfied, its left-hand side has to be positive, which is equivalent to

$$
\frac{1}{\bar{r}_{0}}>\frac{2 t_{n, 0}-t_{n, 1}}{2 t_{n, 0}\left(t_{n, 1}+1\right)}
$$

In particular, the above inequality is satisfied provided that

$$
\begin{aligned}
& 2 t_{n, 0}-t_{n, 1}<0 \text { or } 2 t_{n, 0}-t_{n, 1} \geq 0 \text { and } \\
& \bar{r}_{0}<\frac{2 t_{n, 0}\left(t_{n, 1}+1\right)}{2 t_{n, 0}-t_{n, 1}}=: k_{n}
\end{aligned}
$$

Suppose (7b) is satisfied. Then, (7a) can be further simplified to the following form.

$$
\left(\bar{r}_{0}-1\right)\left[\left(t_{n, 1}-t_{n, 0}\right) \bar{r}_{0}-\left(2 t_{n, 1}\left(t_{n, 1}-t_{n, 0}\right)-t_{n, 0}\right)\right]<0 .
$$

Consequently, the inequality in (7a) holds true provided that (7b) and

$$
1<\bar{r}_{0}<\bar{r}_{+, n}, \text { where } \bar{r}_{+, n}:=2 t_{n, 1}-\frac{t_{n, 0}}{t_{n, 1}-t_{n, 0}}
$$

is satisfied. In fact, only (7c) is needed to imply that (7a) holds true. To see this, it suffices to show that $\bar{r}_{+, n} \leq k_{n}$ whenever $t_{n, 0}<t_{n, 1}<2 t_{n, 0}$, which can be verified by direct calculation. For (7c) to be feasible, it requires that

$$
\bar{r}_{+, n}>1 \text {, or, equivalently, } 2\left(t_{n, 1}-t_{n, 0}\right)>1 \text {. }
$$

We are now in a position to state the conditions for the existence/nonexistence of such special type of period $n$ points.

Proposition 2.4 Consider the existence of a period $n$ solution of the map $\boldsymbol{F}$ for which the epidemic occurs once every $n$ years.

(i) For $n=2$, suppose

$$
\left(\frac{4 c_{1}}{\left(1+c_{1}\right)^{2}}\right) c_{2}+\left(\frac{2}{1+c_{1}}\right) c_{3}>1
$$

Then, the corresponding (7d) is satisfied and the above-mentioned period two points exist whenever $1<\bar{r}_{0}<\bar{r}_{+, 2}$. Moreover, for each $0<c_{1}<1$, there exist feasible $c_{2}$ and $c_{3}$ such that (8a) is satisfied.

(ii) For $n=3$, suppose

$$
\left(\frac{2\left(1+2 c_{1}^{3}\right)}{\left(1+c_{1}+c_{1}^{2}\right)^{2}}\right) c_{2}+\left(\frac{2 c_{1}}{1+c_{1}+c_{1}^{2}}\right) c_{3}>1 .
$$


Then, the associated (7d) is satisfied and the above-mentioned period three points exist whenever $1<\bar{r}_{0}<\bar{r}_{+, 3}$. In particular, there exists a $c_{e, 3}<1$ such that for $0<c_{1}<c_{e, 3}$ (resp., $c_{1} \geq c_{e, 3}$ ) the set of parameters $\left(c_{2}, c_{3}\right)$ satisfying $(8 \mathrm{~b})$ is nonempty (resp., empty).

(iii) For $n \geq 4$, no period $n$ points of this special type exist.

Proof To complete the proof of the proposition, it suffices to find the conditions for which (7d) holds true. To this end, using (5b) and (5d), we write $2\left(t_{n, 1}-t_{n, 0}\right)$ as $a_{n} c_{2}+b_{n} c_{3}$. Then,

$$
a_{n}=2\left(\frac{c_{1}^{n-2}\left(1-c_{1}\right)}{1-c_{1}^{n}}+\left(s_{n, 1}-s_{n, 0}\right)\left(1-c_{1}\right)\right),
$$

and

$$
b_{n}=\frac{2 c_{1}^{n-2}\left(1-c_{1}\right)}{1-c_{1}^{n}}=2 c_{1}^{n-2}\left(\sum_{k=0}^{n-1} c_{1}^{k}\right)^{-1}
$$

Note that

$$
\begin{aligned}
s_{n, 1}-s_{n, 0} & =\frac{1}{\left(1-c_{1}^{n}\right)^{2}}\left\{(n-2) c_{1}^{n-3}+2 c_{1}^{2 n-3}-(n-1) c_{1}^{n-2}-c_{1}^{2 n-2}\right\} \\
& =\frac{1}{\left(1-c_{1}^{n}\right)^{2}}\left\{(n-2) c_{1}^{n-3}\left(1-c_{1}\right)-c_{1}^{n-2}\left(1-c_{1}^{n-1}\right)+c_{1}^{2 n-3}\left(1-c_{1}\right)\right\} \\
& =\frac{\left(1-c_{1}\right)}{\left(1-c_{1}^{n}\right)^{2}}\left\{(n-2) c_{1}^{n-3}+c_{1}^{2 n-3}-c_{1}^{n-2} \sum_{k=0}^{n-2} c_{1}^{k}\right\} .
\end{aligned}
$$

Using the above equality, $a_{n}$ can be further simplified. Specifically,

$$
\begin{aligned}
a_{n}= & \frac{2}{\left(1-c_{1}^{n}\right)^{2}} \times\left\{c_{1}^{n-2}\left(1-c_{1}\right)\left(1-c_{1}^{n}\right)+\right. \\
& \left.\left(1-c_{1}\right)^{2}\left((n-2) c_{1}^{n-3}+c_{1}^{2 n-3}-c_{1}^{n-2} \sum_{k=0}^{n-2} c_{1}^{k}\right)\right\} \\
= & {\left[2(n-2) c_{1}^{n-3}+4 c_{1}^{2 n-3}\right]\left(\sum_{k=0}^{n-1} c_{1}^{k}\right)^{-2} . }
\end{aligned}
$$

Moreover, we have, via (9a) and (9b), that (7d) becomes (8a) and (8b), for $n=2$ and 3 , respectively. Since $b_{2}>1$ for all $0<c_{1}<1$ and $a_{3}>1$ for $0<c_{1}<c_{e, 3}$, see Remark 2.2(i) for more details, we conclude that there are parameter regions for which these special types of period two or three points exist. We have just completed the proof of the assertions in (ii) and (iii). We next show that the assertion in (iii) holds true. 
Suppose

$$
a_{n}<1 \text { for all } n \geq 4 \text { and } 0 \leq c_{1} \leq 1 .
$$

Note that $b_{n}<1$ for all $n \geq 3$. Then, the equality in (7d) means that $a_{n} c_{2}+b_{n} c_{3}>1$, which, in turn, implies that $c_{2}+c_{3}>1$, for all $n \geq 4$ and $0 \leq c_{1} \leq 1$, a contradiction to the assumption that $c_{2}+c_{3} \leq 1$. Hence, to complete the third assertion of the theorem, it remains to show that (10) holds true. For $n \geq 5$, it is easy to see that, by expanding $\left(\sum_{k=0}^{n-1} c_{1}^{k}\right)^{2}$, there are no less than or equal to $2(n-2)$ terms containing $c_{1}^{k}$, $0 \leq k \leq n-3$. Moreover, the total number of the terms containing $c_{1}^{k}, n-3<k \leq$ $2 n-3$, is greater than 4 . Hence, for $n \geq 5$, (10) holds as claimed. For $n=4$, it is obvious that

$$
\begin{aligned}
4 c_{1}+4 c_{1}^{5} & <\left(1+c_{1}\right)^{2}+2\left(1+c_{1}\right)\left(c_{1}^{2}+c_{1}^{3}\right)+\left(c_{1}^{2}+c_{1}^{3}\right)^{2} \\
& =\left(\sum_{k=0}^{3} c_{1}^{k}\right)^{2} .
\end{aligned}
$$

We just proved the third assertion of the theorem. Now, (8a) and (8b) can be directly obtained from (9a) and (9b). For $n=2$, clearly $b_{2}>1$ and $a_{2}<1$. Hence, for each $0<c_{1}<1$, there exist feasible $c_{2}$ and $c_{3}$ such that (8a) is satisfied. For $n=3, b_{3}<1$, $a_{3}=\frac{2\left(1+2 c_{1}^{3}\right)}{1+c_{1}+c_{1}^{2}}$. Some elementary calculations would yield that there exists a $c_{e, 3}$ such that $a_{3}>1$ (resp. $\left.<1\right)$ whenever $c \in\left(0, c_{e, 3}\right)$ (resp. $\left.\left(c_{e, 3}, 1\right)\right)$. Hence, we have just completed the proof the theorem.

Remark 2.2 (i) Numerically, $c_{e, 3} \approx 0.3509168319$. The notation $c_{e, 3}$ is so set up that its subscripts $e$ and 3 represent the existence of period three points. Similar notations will be employed throughout the paper. The equations in (8a) and (8b) are to be denoted by $\ell_{e, 2}$ and $\ell_{e, 3}$, respectively. For fixed $c_{1}$, we illustrate the parameter region in the $c_{2}-c_{3}$ plane for which the existence of period 2 or 3 points exists in Fig. 1.

(ii) The intersection point $I_{2,3}$ of $\ell_{e, 2}$ and $\ell_{e, 3}$ lies above the line $\ell\left(c_{2}+c_{3}=1\right)$. Here $I_{2,3}=\left(\frac{\left(c_{1}+1\right)\left(c_{1}^{2}+c_{1}+1\right)}{2\left(1-c_{1}\right)\left(1+2 c_{1}\right)}, \frac{1-3 c_{1}^{2}-4 c_{1}^{3}}{2\left(1-c_{1}\right)\left(1+2 c_{1}\right)}\right)$. The verification of the above is cumbersome but elementary. This implies that the coexistence of such period two and three points is impossible.

(iii) The lines $\ell$ and $\ell_{e, 2}$ intersect at $\left(\frac{1+c_{1}}{2}, \frac{1-c_{1}}{2}\right)$.

(iv) Using (ii) and (iii), we also arrive at the conclusion that $c_{2}>c_{3}$ is a necessary condition for the existence of such period three points.

(v) Clearly, there are three types of period three points, depending on the number of the infected population being zero at the year end in a three-year span. We shall use the notations $3(+,+,+), 3(0,+,+), 3(0,0,+)$ to distinguish one from the other. In particular, the special type of period three points described in Proposition 2.4 is of $3(0,0,+)$ type. Similar notations are to be used for period $n$ points if needed. 
Fig. $1 \ell: c_{2}+c_{3}=1$,

$\ell_{e, 2}: \frac{4 c_{1}}{\left(1+c_{1}\right)^{2}} c_{2}+\frac{2}{\left(1+c_{1}\right)} c_{3}=1$

and $\ell_{e, 3}: \frac{2\left(1+2 c_{1}^{3}\right)}{\left(1+c_{1}+c_{1}^{2}\right)^{2}} c_{2}$

$+\frac{2 c_{1}}{1+c_{1}+c_{1}^{2}} c_{3}=1$ with

$c_{1}=0.1$. The green (resp., blue) region represents the parameter region for the existence of period two (resp., three) points mentioned in Proposition 2.4. These two regions do not intersect with each other (colour figure online)

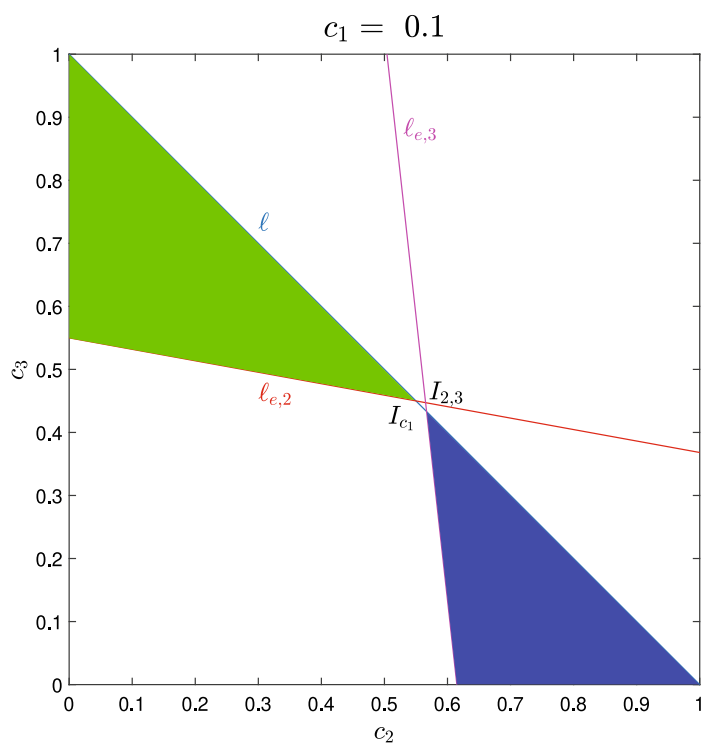

Our first main result deals with the stability of the fixed point. For $\bar{r}_{0}>1$, let $\left(x_{*}, y_{*}, p_{*}\right)$, be defined as in Remark 2.1. The Jacobian matrix of $\mathbf{F}$ at $\left(x_{*}, y_{*}\right)$ has the following form.

$$
\mathbf{F}^{\prime}\left(x_{*}, y_{*}\right)=\mathbf{C}+b_{*, 1}\left(\begin{array}{ll}
c_{2} & c_{2} \\
c_{3} & c_{3}
\end{array}\right)
$$

Here $\mathbf{C}$ is defined in (1b) and

$$
\begin{aligned}
b_{*, 1} & =\left.\frac{\partial p_{n}}{\partial x_{n}}\right|_{\left(x_{n}, y_{n}\right)=\left(x_{*}, y_{*}\right)} \\
& =\left.\frac{\partial p_{n}}{\partial y_{n}}\right|_{\left(x_{n}, y_{n}\right)=\left(x_{*}, y_{*}\right)}=-2\left(1-\frac{\bar{r}_{0}}{2} p_{*}\right)^{2} .
\end{aligned}
$$

We have used the fact, via (1c), that $p_{*}=\frac{2}{\bar{r}_{0}}\left(1-\frac{1}{\bar{r}_{0}\left(1-x_{*}-y_{*}\right)}\right)$ to justify the last equality in (11a). Consequently,

$$
-2<b_{*, 1}<0
$$

Some direct calculations would yield that the characteristic polynomial of $F^{\prime}\left(x_{*}, y_{*}\right)$ is $\lambda^{2}-\alpha_{1} \lambda+\beta_{1}=0$, where

$$
\alpha_{1}=2 c_{1}+\left(c_{2}+c_{3}\right) b_{*, 1} \text { and } \beta_{1}=c_{1}^{2}-b_{*, 1}\left(c_{2}\left(1-c_{1}\right)-c_{1} c_{3}\right) \text {. }
$$


Define

$$
b_{\Delta, 1}=-\frac{4 c_{2}\left(1-c_{1}\right)}{\left(c_{2}+c_{3}\right)^{2}} .
$$

Then, $\lambda_{ \pm}$are complex (resp., real) roots whenever $b_{\Delta, 1}<b_{*, 1}<0$ (resp., $b_{*, 1} \leq$ $b_{\Delta, 1}$ ). We next compute the equivalent conditions for the following three inequalities $\lambda_{+}<1, \lambda_{-}>-1$ and $\left(\lambda_{+}\right)\left(\lambda_{-}\right)<1$. Those equivalent conditions are, respectively, $b_{*, 1}<\frac{1-c_{1}}{2 c_{2}+c_{3}}$

$$
b_{*, 1}<\frac{-\left(1+c_{1}\right)^{2}}{2 c_{1} c_{2}+\left(1+c_{1}\right) c_{3}}=: b_{r, 1}
$$

and

$$
c_{1}^{2}-b_{*, 1}\left(c_{2}-\left(2 c_{2}+c_{3}\right) c_{1}\right)<1 .
$$

Since $b_{*, 1}<0<\frac{1-c_{1}}{2 c_{2}+c_{3}}$, the inequality $\lambda_{+}<1$ is satisfied for all feasible parameters. Hence, if $b_{*, 1} \leq b_{\Delta, 1}$, then $\lambda_{ \pm}$are real roots and so $\lambda_{-} \leq \lambda_{+}$. Consequently, if $b_{r, 1}<b_{*, 1} \leq b_{\Delta, 1}$, we have that $-1<\lambda_{-} \leq \lambda_{+}<1$, a sufficient condition for the fixed point $\left(x_{*}, y_{*}\right)$ to be locally stable. Suppose $c_{1}<\frac{c_{2}}{2 c_{2}+c_{3}}$. Define $b_{c, 1}$ as

$$
b_{c, 1}:=\frac{-\left(1-c_{1}^{2}\right)}{c_{2}-\left(2 c_{2}+c_{3}\right) c_{1}} .
$$

Then, (11e) is equivalent to $b_{*, 1}>b_{c, 1}$. On the other hand, if $c_{1} \geq \frac{c_{2}}{2 c_{2}+c_{3}}$, then (11e) is also satisfied and so is the inequality $\left(\lambda_{+}\right)\left(\lambda_{-}\right)<1$. We are now in a position to summarize the stability of the fixed point.

Theorem 2.1 (I) If one of the following conditions holds true, then the fixed point $\left(x_{*}, y_{*}\right)$ of the map $F$ is locally stable for all $\bar{r}_{0}$.

(I-a) $b_{r, 1}<b_{*, 1} \leq b_{\Delta, 1}$.

(I-b) $c_{1} \geq \frac{c_{2}}{2 c_{2}+c_{3}}$ and $b_{*, 1}>b_{r, 1}$.

(I-c) $c_{1}<\frac{c_{2}}{2 c_{2}+c_{3}}$ and $b_{*, 1}>\max \left\{b_{\Delta, 1}, b_{c, 1}\right\}$.

(I-d) $\max \left\{b_{r, 1}, b_{c, 1}\right\}<-2$.

(II) For $0<c_{1}<1$, we denote by $A_{r, c_{1}}$ (resp., $A_{c, c_{1}}$ ) the interception point of the lines $b_{r, 1}=-2$ (resp., $b_{c, 1}=-2$ ) and $c_{2}+c_{3}=1$. Moreover, let $I_{r, c_{1}}$ (resp, $I_{c, c_{1}}$ ) be the $c_{3}$ (resp., $c_{2}$ ) intercept of the line $b_{r, 1}=-2\left(\right.$ resp., $\left.b_{c, 1}=-2\right)$. Then, the $\left(x_{*}, y_{*}\right)$ is locally stable provided that the parameter point $\left(c_{2}, c_{3}\right)$ is in the interior of one of the following two regions.

(II-a) The quadrilateral $O I_{c, 2-\sqrt{3}} A_{r, c_{1}} I_{r, c_{1}}$, see Fig. 2a. Here $2-\sqrt{3} \leq c_{1}<1$. (II-b) The pentagon $O I_{c, c_{1}} A_{c, c_{1}} A_{r, c_{1}} I_{r, c_{1}}$, see Fig. $2 b$. Here $0<c_{1}<2-\sqrt{3}$.

(III) For $\bar{r}_{0}$ sufficiently large, the corresponding fixed point is locally stable. 


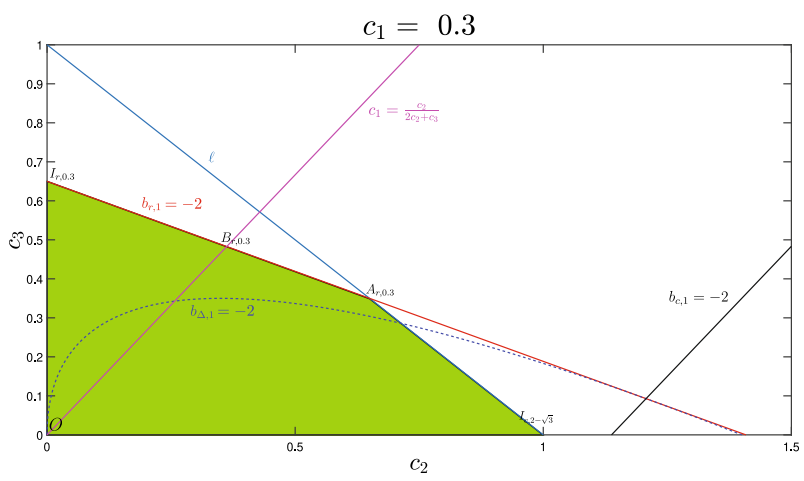

(a) The stable (green) region of the fixed point in the $c_{2}-c_{3}$ plane. Here $2-\sqrt{3} \leq$ $c_{1} \leq 1$. Its boundaries are the $c_{2}$ and $c_{3}$ axes, the lines $\ell$ and $b_{r, 1}=-2$. Here $b_{r, 1}=$ -2 and $\ell_{e, 2}$ represent the same line

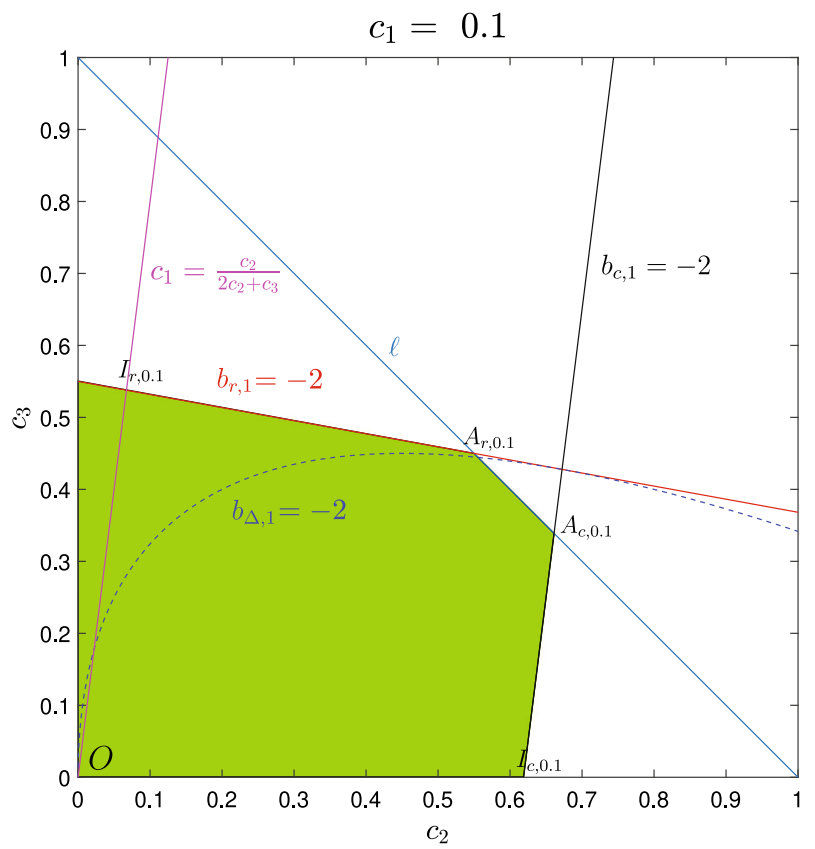

(b) The stable (green) region of the fixed point in the $c_{2}-c_{3}$ plane. Here $0<c_{1}<$ $2-\sqrt{3}$. Its boundaries are the $c_{2}$ and $c_{3}$ axes, the lines $b_{c, 1}=-2, \ell$ and $b_{r, 1}=-2$.

Fig. 2 Typically stable regions, described in Theorem 2.1-(II), in the $c_{2}-c_{3}$ plane 
Proof For condition (I-a) to be valid, we first show that

$$
b_{\Delta, 1}>b_{r, 1} \text { for all feasible } c_{i}, i=1,2,3 \text {. }
$$

To see this, we get that $b_{\Delta, 1}>b_{r, 1}$ if and only if $g_{1}\left(c_{1}\right)>0$. Indeed,

$$
\begin{aligned}
g_{1}\left(c_{1}\right) & :=\left(3 c_{2}+c_{3}\right)^{2} c_{1}^{2}-2\left(c_{2}-c_{3}\right)\left(3 c_{2}+c_{3}\right) c_{1}+\left(c_{2}-c_{3}\right)^{2} \\
& =\left(\left(3 c_{2}+c_{3}\right) c_{1}+c_{2}-c_{3}\right)^{2}>0
\end{aligned}
$$

The proof that condition (I-a) implies the stability of $\left(x_{*}, y_{*}\right)$ was provided on the paragraph in between (11e) and (11f). If $c_{1} \geq \frac{c_{2}}{2 c_{2}+c_{3}}$, then $b_{\Delta, 1}>0$, and so, $b_{*, 1}<b_{\Delta, 1}$ due to the fact that $b_{*, 1}<0$ as indicated in (11b). As a result, $\lambda_{ \pm}$are real roots. It then follows from (I-a) that $\left(x_{*}, y_{*}\right)$ is stable as asserted. On the other hand, if $c_{1}<\frac{c_{2}}{2 c_{2}+c_{3}}$, then $\left(x_{*}, y_{*}\right)$ is stable as long as $b_{*, 1}>b_{c, 1}$ holds. Hence, if condition (Ic) holds, then $\left(x_{*}, y_{*}\right)$ is stable as claimed. We next show that if (I-d) is satisfied, then $\left(x_{*}, y_{*}\right)$ is stable. To see this, we break $b_{\Delta, 1}$ into the following cases. Let $b_{\Delta, 1} \geq-2$. It follows from (11a) that $b_{*, 1}>\max \left\{b_{r, 1}, b_{c, 1}\right\}$. Now, suppose $b_{*, 1}>b_{\Delta, 1}$. Then, $\left(x_{*}, y_{*}\right)$ is stable by (I-b) and (I-c). On the other hand, if $b_{*, 1} \leq b_{\Delta, 1}$, then the stability of $\left(x_{*}, y_{*}\right)$ is guaranteed by (I-a). For the case that $b_{\Delta, 1}<-2$, we see that $b_{*, 1}>\max \left\{b_{\Delta, 1}, b_{r, 1}, b_{c, 1}\right\}$. Hence, $\left(x_{*}, y_{*}\right)$ is also stable by (I-b) and (I-c). We just completed the proof concerning (I-d).

We now move to prove the second part of the theorem. In fact, if the algebraic condition (I-d) is converted into the geometric region in the $\left(c_{2}, c_{3}\right)$ plane, then the shape of corresponding region has two types, which are described in (II-a) and (II-b). To see this, we first note that $b_{r, 1} \leq-2$ and $b_{c, 1} \leq-2$ are, respectively, equivalent to the following inequalities.

$$
\left(\frac{4 c_{1}}{\left(1+c_{1}\right)^{2}}\right) c_{2}+\left(\frac{2}{1+c_{1}}\right) c_{3} \leq 1
$$

and

$$
\left(\frac{1-c_{1}^{2}}{2\left(1-2 c_{1}\right)}\right) c_{2}-\left(\frac{2 c_{1}}{1-c_{1}^{2}}\right) c_{3} \leq 1
$$

It should be mentioned that the $c_{2}$ intercept of the line $b_{c, 1}=-2$ is equal to one provided that $c_{1}=2-\sqrt{3}$. It is then easy to see that the $c_{2}$-intercept is greater than or equal to one (resp., smaller than one) provided that $c_{1}<2-\sqrt{3}$ (resp., $2-\sqrt{3}<c_{1}<\frac{1}{2}$ ). For $c_{1} \geq \frac{1}{2}, b_{c, 1}$ is always less than or equal to -2 since both the $c_{2}$ and $c_{3}$ intercepts are negative. We just completed the proof of the second part of the theorem.

To prove the last part of the theorem, we see that $\lim _{\bar{r}_{0} \rightarrow \infty} b_{*, 1}=0$. It then follows from (11c) that $\lambda_{ \pm}$are complex roots provided that $\bar{r}_{0}$ is sufficiently large. Upon using (11e), we get that $\left|\lambda_{ \pm}\right|<1$ whenever $\bar{r}_{0}$ is sufficiently large. 
Remark 2.3 (a) Using Remark 2.1, we have that

$$
b_{*, 1}=-8\left(\sqrt{\left(2 c-\bar{r}_{0}\right)^{2}+8 c}-\left(2 c-\bar{r}_{0}\right)\right)^{-2},
$$

where $c$ is given in (3a). Some direct calculations would yield that $\frac{\partial}{\partial \bar{r}_{0}}\left(b_{*, 1}\right)=$ $-\left(2 c-\bar{r}_{0}\right)\left(\left(2 c-\bar{r}_{0}\right)^{2}+8 c\right)^{\frac{-1}{2}}+1>0$, for all $c>0$ and $\frac{\partial}{\partial c}\left(b_{*, 1}\right)=2((2 c-$ $\left.\left.\bar{r}_{0}\right)^{2}+8 c\right)^{\frac{-1}{2}}\left(\left(2 c-\bar{r}_{0}\right)+2-\sqrt{\left(2 c-\bar{r}_{0}\right)^{2}+8 c}\right)<0$ for all $\bar{r}_{0}>1$. Consequently, $b_{*, 1}$ is increasing in $\bar{r}_{0}$ and decreasing in $c_{1}$. Moreover, it is easy to see that $\lim _{\bar{r}_{0} \rightarrow 1^{+}} b_{*, 1}=\lim _{c_{1} \rightarrow 1^{-}} b_{*, 1}=-2 \leq b_{*, 1}$ for all feasible $c_{1}$ and $\bar{r}_{0}$. This amounts to saying that one may chose suitable parameters so that $b_{*, 1}$ can be arbitrarily close to -2 from the right.

(b) The assumptions on the (II-a) and (II-a) of Theorem 2.1 are only sufficient conditions for $\left(x_{*}, y_{*}\right)$ to be stable. To see this, let $c_{1}=c_{2}=0, \bar{r}_{0}=2$ and $c_{3}>\frac{1}{2}$, the corresponding $b_{*, 1}, b_{\Delta, 1}$ and $b_{r, 1}$ are, respectively, $\frac{-2}{\left(\sqrt{1+c_{3}^{2}}+1-c_{3}\right)^{2}}, 0$, and $-\frac{1}{c_{3}}$. Hence, $b_{\Delta, 1}>b_{*, 1}>b_{r, 1}>-2$. By choosing $c_{1}$ and $c_{2}$ sufficiently small and $\bar{r}_{0}$ sufficiently close to 2 , we have, via Theorem 2.1-(I-b), that the corresponding fixed point is locally stable.

In the following, we state a sufficient condition for the stability of the special type of period two point described in Proposition 2.4.

Theorem 2.2 Suppose (8a) is satisfied and so $\bar{r}_{+, 2}>1$. For $0<c_{1}<1$, if in addition, $c_{2}$ and $c_{3}$ satisfy the following inequality

$$
2\left(1-c_{1}^{2}+2 c_{1}^{3}\right) c_{2}+2\left(c_{1}+c_{1}^{3}\right) c_{3} \leq\left(1+c_{1}^{2}\right)^{2},
$$

then the period two points of $2(0,+)$ type is locally stable for all $\bar{r}_{0} \in\left(1, \bar{r}_{+, 2}\right)$. Pictorially, the green (stable) region as shown in Fig. 3 is where $\left(c_{2}, c_{3}\right)$ satisfies both (8a) and (12a).

Proof For $n=2$, let $\left(x_{0,0,2}, y_{0,0,2}, \bar{p}_{2}\right)$ and $\left(x_{1,0,2}, y_{1,0,2}, 0\right)$ be the period two points of $2(0,+)$ type. Then, the local stability of such period two points is determined by the size of the spectral radius of the product of two Jacobian matrices $\mathbf{F}^{\prime}\left(x_{0,0,2}, y_{0,0,2}\right)$ and $\mathbf{F}^{\prime}\left(x_{1,0,2}, y_{1,0,2}\right)$, which has the following form

$$
\left(\begin{array}{cc}
c_{1}^{2}+c_{2} b_{*, 2} & c_{1} c_{2} b_{*, 2} \\
2 c_{1}\left(1-c_{1}\right)+c_{3} b_{*, 2} & c_{1}^{2}+c_{1} c_{3} b_{*, 2}
\end{array}\right):=\mathbf{F}_{2}
$$

where $b_{*, 2}=\frac{-2}{\bar{r}_{0}^{2}\left(1-x_{0,0,2}-y_{0,0,2}\right)^{2}}$. Note that $-2 \leq b_{*, 2} \leq \frac{-2}{\bar{r}_{0}^{2}}$. The subscript 2 of $b_{*, 2}$ indicates the quantity is associated with this special type of period two points. 


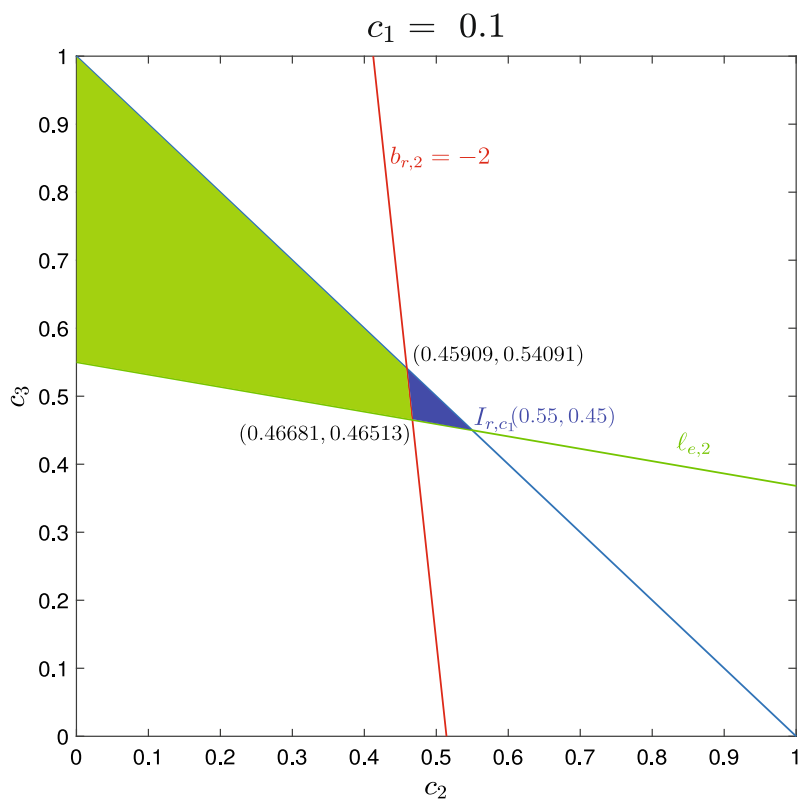

Fig. 3 Stable (green) region for the period two points. Here $1<\bar{r}_{0}<\bar{r}_{+, 2}$. Its boundaries are $\ell_{e, 2}$, equality in (8a), $b_{r, 2}=-2$, equality in (12a), $\ell$ and the $c_{3}$-axis. For $\bar{r}_{0}>\bar{r}_{+, 2}$, the period two points do not exist (colour figure online)

The corresponding characteristic polynomial of the matrix $\mathbf{F}_{2}$ has the following form $\lambda^{2}-\alpha_{2} \lambda+\beta_{2}=0$, where

$$
\alpha_{2}=2 c_{1}^{2}+\left(c_{2}+c_{1} c_{3}\right) b_{*, 2}
$$

and

$$
\beta_{2}=c_{1}^{4}-c_{1}^{2}\left(\left(1-2 c_{1}\right) c_{2}-c_{1} c_{3}\right) b_{*, 2}=: c_{1}^{4}-c_{1}^{2} d_{1} b_{*, 2}
$$

Let $\Delta_{2}=\alpha_{2}^{2}-4 \beta_{2}$. If $\Delta_{2}<0$ and $\beta_{2}<1$ or $\Delta_{2} \geq 0$ and $1+\beta_{2}>\left|\alpha_{2}\right|$, then such special type of period two points is locally stable. Since (8a) is satisfied, $\left(c_{2}, c_{3}\right)$ lies in the triangular region for which its three vertices are $(0,1),\left(0, \frac{1+c_{1}}{2}\right)$ and $\left(\frac{1+c_{1}}{2}, \frac{1-c_{1}}{2}\right)$. It is clear that the maximum of $d_{1}$ as defined in (12c) occurs at $\left(\frac{1+c_{1}}{2}, \frac{1-c_{1}}{2}\right)$. Consequently, $d_{1}<\frac{1}{2}\left(1-2 c_{1}-c_{1}^{2}\right)$ for all $c_{1} \in(0,1)$. Hence, $\beta_{2}<$ $c_{1}^{4}+c_{1}^{2}\left(1-2 c_{1}-c_{1}^{2}\right)=c_{1}^{2}\left(1-2 c_{1}\right)<1$ regardless of the sign of $\Delta_{2}$. The verification of the sign of $1+\beta_{2}-\alpha_{2}$ is obvious since $1+\beta_{2}-\alpha_{2}=\left(1-c_{1}^{2}\right)^{2}-\left(\left(1+c_{1}^{2}-2 c_{1}^{3}\right) c_{2}+\right.$ $\left.\left(c_{1}-c_{1}^{3}\right) c_{3}\right) b_{*, 2}$, which is clearly positive for all $0<c_{1}<1$ regardless of the sign of $\Delta_{2}$. To complete the proof of the theorem, it remains to show that $1+\beta_{2}+\alpha_{2}>0$ whenever $(8 \mathrm{a})$ and $(12 \mathrm{a})$ are satisfied. Let $d_{2}:=\left(1-c_{1}^{2}+2 c_{1}^{3}\right) c_{2}+\left(c_{1}+c_{1}^{3}\right) c_{3}$. Then, 
$1+\beta_{2}+\alpha_{2}=\left(1+c_{1}^{2}\right)^{2}+d_{2} b_{*, 2}$, which is greater than zero whenever

$$
b_{*, 2}>-\frac{\left(1+c_{1}^{2}\right)^{2}}{d_{2}}=: b_{r, 2} .
$$

The above inequality is satisfied provided that $b_{r, 2} \leq-2$, or equivalently, (12a) holds true. Clearly, the $c_{3}$ (resp., $c_{2}$ ) intercept of the line $b_{r, 2}=-2$ is greater (resp., less) than one. Moreover, the point $I_{r, c_{1}}=\left(\frac{1+c_{1}}{2}, \frac{1-c_{1}}{2}\right)$, which is the intersection of the line $c_{2}+c_{3}=1$ and the line defined by the equality presented in (8a), and the origin are on the opposite side of the half planes, determined by $b_{r, 2}=-2$. Hence, the green region in Fig. 3 is a stable region. We have completed the proof of the theorem.

Note that $I_{r, c_{1}}$ and the origin are on the opposite side of the half planes, divided by $b_{r, 2}=-2$. However, it should be pointed out that for the parameters chosen from the blue region, see Fig. 3, the corresponding period two points are still possibly be stable depending on the range of $\bar{r}_{0}$. We next investigate the stability of the period three points described in Proposition 2.4 and the possibility of bistable states. The numerical simulations suggest the that the existence of bistable states can be observed for certain ranges of the parameters. We then aim to provide inside as to what range of parameters would generate those bistable states.

Theorem 2.3 (I) Let $c_{e, 3}$ be defined as described in Remark 2.2(i). Let $b_{r, 3}$ be defined as follows.

$$
b_{r, 3}:=\frac{-\left(1+2 c_{1}^{3}+c_{1}^{6}\right)}{\left(2 c_{1}-c_{1}^{2}-c_{1}^{4}+2 c_{1}^{5}\right) c_{2}+\left(c_{1}^{2}+c_{1}^{5}\right) c_{3}} .
$$

Consequently, the inequality $b_{r, 3} \leq-2$ has the following form.

$$
2\left(2 c_{1}-c_{1}^{2}-c_{1}^{4}+2 c_{1}^{5}\right) c_{2}+2\left(c_{1}^{2}+c_{1}^{5}\right) c_{3} \leq\left(1+2 c_{1}^{3}+c_{1}^{6}\right) .
$$

The stability region of the period three points of $3(0,0,+)$ type is addressed in the following.

(I-a) There exists a $c_{s_{1}, 3}\left(<c_{e, 3}\right)$ such that if $0<c_{1} \leq c_{s_{1}, 3}$, then the existence of such period three points implies its stability for any $\bar{r}_{0} \in\left(1, \bar{r}_{+, 3}\right)$. Here $c_{s_{1}, 3}$ is the number so obtained that the $c_{2}$-intercept of the line $b_{r, 3}=-2$ is equal to 1 when $c_{1}=c_{s_{1}, 3}$. Numerically, $c_{s_{1}, 3} \approx 0.31949$. A stable region in the $c_{2}-c_{3}$ plane for such range of $c_{1}$ is depicted in the blue region in Fig. 1.

(I-b) There exists a $c_{s_{2}, 3}$ such that, for $c_{s_{1}, 3}<c_{1}<c_{s_{2}, 3}$ and $\bar{r}_{0} \in\left(1, \bar{r}_{+, 3}\right)$, its corresponding stability region, determined by (8b) and (13b), is nonempty. Here $c_{s_{2}, 3}$ is the number so obtained that the three lines $\ell$, see Fig. $1, b_{r, 3}=-2$, and $\ell_{e, 3}$ have a common intersection when $c_{1}=c_{s_{2}, 3}$. Numerically, $c_{s_{2}, 3} \approx$ 0.33747 . A stability region placed on the $c_{2}-c_{3}$ plane is illustrated as the green region in Fig. 6.

II The map $\boldsymbol{F}$ exhibits $(1,3(0,0,+))$ stability for any $\bar{r}_{0} \in\left(1, \bar{r}_{+, 3}\right)$ if one of the following three conditions is fulfilled. 
(II-a) $0<c_{1}<2-\sqrt{3}, c_{2}$ and $c_{3}$ satisfy (8b) and $b_{c, 1} \leq-2$, see the green region in Fig. 4.

(II-b) $2-\sqrt{3} \leq c_{1} \leq c_{s_{1}, 3}, c_{2}$ and $c_{3}$ satisfy (8b), see the green region in Fig. 5 .

(II-c) $c_{s_{1}, 3}<c_{1}<c_{s_{2}, 3}, c_{2}$ and $c_{3}$ satisfy (8b) and (13b), see the green region in Fig. 6.

In particular, for parameters chosen from the region described in (II-b), the existence of such period three points implies $(1,3(0,0,+))$-stability.

Proof For $n=3$, let $\left(x_{0,0,3}, y_{0,0,3}, \bar{p}_{3}\right),\left(x_{1,0,3}, y_{1,0,3}, 0\right)$ and $\left(x_{2,0,3}, y_{2,0,3}, 0\right)$ be the period three points of $3(0,0,+)$ type. Then, the product of Jacobian matrices $\mathbf{F}^{\prime}$ at those three period three points, respectively, has the following form

$$
\left(\begin{array}{cc}
c_{1}^{3}+c_{1} c_{2}\left(2-c_{1}\right) b_{*, 3} & c_{1}^{2} c_{2} b_{*, 3} \\
3 c_{1}^{2}\left(1-c_{1}\right)+c_{1} c_{3}\left(2-c_{1}\right) b_{*, 3} & c_{1}^{3}+c_{1}^{2} c_{3} b_{*, 3}
\end{array}\right)=: \mathbf{F}_{3},
$$

where $-2 \leq b_{*, 3}=\frac{-2}{\bar{r}_{0}^{2}\left(1-x_{0,0,3}-y_{0,0,3}\right)^{2}} \leq-\frac{2}{\bar{r}_{0}^{2}}$. Some direct calculation would yield that the characteristic polynomial of $\mathbf{F}_{3}$ has the following form $\lambda^{2}-\alpha_{3} \lambda+\beta_{3}=0$, where

$$
\alpha_{3}=2 c_{1}^{3}+\left(c_{1}\left(2-c_{1}\right) c_{2}+c_{1}^{2} c_{3}\right) b_{*, 3}=: 2 c_{1}^{3}+d_{3} b_{*, 3}
$$

and

$$
\beta_{3}=c_{1}^{6}+c_{1}^{4}\left(\left(2 c_{1}-1\right) c_{2}+c_{1} c_{3}\right) b_{*, 3}=: c_{1}^{6}+c_{1}^{4} d_{4} b_{*, 3}
$$

We then follow the similar argument as those in proving the last theorem. To this end, we first show that $\left|\beta_{3}\right|<1$. Using Remark 2.2(i) and (iv), we have that $-c_{2} \leq d_{3} \leq$ $\frac{1}{2} c_{2}$. Hence, $\left|\beta_{3}\right|<c_{1}^{6}+2 c_{1}^{4}<1$ for $c<\frac{1}{2}$. Now,

$$
1+\beta_{3}-\alpha_{3}=1-2 c_{1}^{3}+c_{1}^{6}-\left(c_{2}\left(2 c_{1}-c_{1}^{2}+c_{1}^{4}-2 c_{1}^{5}\right)+\left(c_{1}^{2}-c_{1}^{5}\right) c_{3}\right) b_{*, 3},
$$

which is positive for any $0<c_{1}<1$. Next, we have that $1+\beta_{3}+\alpha_{3}>0$ provided that $b_{*, 3}>b_{r, 3}$. The above inequality is satisfied as long as $-2 \geq b_{r, 3}$ or, equivalently, (13b) holds true. It is easy to check that the $c_{3}$-intercept of the line $b_{r, 3}=-2$ is greater than 1 for $0<c_{1} \leq c_{e, 3}\left(<\frac{1}{2}\right)$. The $c_{2}$-intercept of the line $b_{r, 3}=-2$ is greater than or equal to 1 if and only if

$$
1-4 c_{1}+2 c_{1}^{2}+2 c_{1}^{3}+2 c_{1}^{4}-4 c_{1}^{5}+c_{1}^{6}=: g\left(c_{1}\right)>0 .
$$

It is easy to set that $g$ is decreasing on $\left(0, \frac{1}{3}\right)$. Since $g(0)>0$, and $g\left(\frac{1}{3}\right)<0$, there exists a $c_{s_{1}, 3}$ such that $g\left(c_{s_{1}, 3}\right)=0$ and the corresponding $c_{2}$ intercept is greater than or equal to one (resp., smaller than one) for $c_{1} \in\left(0, c_{s_{1}, 3}\right]$ (resp., $\left.\left(c_{s_{1}, 3}, c_{e, 3}\right)\right)$. In fact, $c_{s_{1}, 3} \approx 0.31949$. Hence, for such range of $c_{1}$, condition (13b) is automatically satisfied for all feasible $c_{2}$ and $c_{3}$. Consequently, the existence of the period three 
points implies its stability. To complete the assertion in (I-b), we need to show that the inequalities ( $8 \mathrm{~b}),(13 \mathrm{~b})$ and $0<c_{2}+c_{3} \leq 1$ have a nonempty (stability) region in the $c_{2}-c_{3}$ plane. To this end, we first show that the slope $m_{e}$ of $\ell_{e, 3}$ is larger than that $m_{r}$ of $b_{r, 3}=-2$ whenever $c_{1}<0.4$. Some direct calculation would yield that

$$
m_{e}=\frac{-\left(1+2 c_{1}^{3}\right)}{c_{1}\left(1+c_{1}+c_{1}^{2}\right)}>m_{r}=\frac{-\left(2-c_{1}-c_{1}^{3}+2 c_{1}^{4}\right)}{c_{1}+c_{1}^{4}}
$$

if and only if $1+c_{1}+c_{1}^{2}-5 c_{1}^{3}+c_{1}^{4}+c_{1}^{5}>0$, which clearly holds for all feasible $c_{1}$. We next compute the intersection $\left(\bar{c}_{2}, \bar{c}_{3}\right)$ of the lines $\ell_{3}$ and $b_{r, 3}=-2$. After some tedious calculation, we have that

$$
\begin{aligned}
& \bar{c}_{2}=\frac{-c_{1}^{7}-2 c_{1}^{6}-c_{1}^{5}-c_{1}^{4}-c_{1}^{3}-c_{1}^{2}+1}{2 c_{1}^{6}+2 c_{1}^{5}-10 c_{1}^{4}+2 c_{1}^{3}+2 c_{1}^{2}+2 c_{1}} \text { and } \\
& \bar{c}_{3}=\frac{3 c_{1}^{8}+4 c_{1}^{7}-5 c_{1}^{6}+3 c_{1}^{2}+2 c_{1}-1}{2 c_{1}^{7}+2 c_{1}^{6}-10 c_{1}^{5}+2 c_{1}^{4}+2 c_{1}^{3}+2 c_{1}^{2}}
\end{aligned}
$$

and that $\bar{c}_{2}+\bar{c}_{3} \leq 1$ if and only if $\left(c_{1}^{2}-c_{1}+1\right)\left(2 c_{1}^{5}+4 c_{1}^{4}-4 c_{1}^{3}-5 c_{1}^{2}-c_{1}+1\right) \geq 0$. Solving $\bar{c}_{2}+\bar{c}_{3}=1$ numerically, we have that $c_{1} \approx 0.33747=: c_{S_{2}, 3}$ or -2.44783 or 1.25025. Clearly, $c_{s_{2}, 3}$ is the only feasible solution. Using all the above information, we arrive at the conclusion that $\bar{c}_{2}+\bar{c}_{3}<1$ (resp., $\geq 1$ ) whenever $c_{1} \in\left(0, c_{s_{2}, 3}\right)$ (resp., $\left.\left[c_{s_{2}, 3}, 1\right]\right)$. Upon using the fact that $0>m_{e}>m_{r}$, we further conclude that the stability region is nonempty (resp., empty) for $c_{1} \in\left(c_{s_{1}, 3}, c_{s_{2}, 3}\right)$ (resp., [ $\left.c_{s_{2}, 3}, c_{e, 3}\right)$ ).

For $0<c_{1}<2-\sqrt{3}$, see Fig. 2b, we have, via Theorem 2.1-(II-b) and Theorem 2.3-(I), that the map $\mathbf{F}$ exhibits $(1,3(0,0,+))$-stability provided that assumptions in (IIa) hold true. Such region, see Fig. 4 , is nonempty provided that the $c_{2}$-intercept of $b_{c, 1}=-2$ is greater than that of $\ell_{3}$. We omit the elementary verification of such claims. Suppose $2-\sqrt{3} \leq c_{1} \leq c_{S_{1}, 3}$. Then, the $c_{2}$-coordinate of $A_{r, c_{1}}=\frac{1+c_{1}}{2}$ and that of the interception point $A_{3, c_{1}}$ of $\ell_{3}$ and $c_{2}+c_{3}=1$ is $\frac{1+c_{1}^{2}+c_{1}^{4}}{2\left(1-c_{1}\right)^{2}\left(1+c_{1}\right)}\left(>\frac{1+c_{1}}{2}\right)$. Hence, $A_{r, c_{1}}$ is to the left of $A_{3, c_{1}}$. It then follows from Theorem 2.1-(II-a) and Theorem 2.3-(I) that the map $\mathbf{F}$ exhibits the $(1,3(0,0,+))$-stability as long as such period three points exist. For $c_{s_{1}, 3}<c_{1}<c_{s_{2}, 3}$, it is clear that the stability of period three points of $3(0,0,+)$ type implies $(1,3(0,0,+))$-stability of the map. We just completed the proof of the theorem.

We conclude the section by mentioning that, for a certain range of the parameters, other type of multistability such as $(3(0,0,+), \mathrm{C})$-stability and $(3(0,0,+), 8)$-stability can be observed numerically. Specifically, the parameters chosen from the blue region in Fig. 4 may exhibit interesting and complicated dynamics as $\bar{r}_{0}$ varies. To see this, we first fix $c_{1}=0.1, c_{2}=0.8$ and $c_{3}=0.2$ and pick two sets of initial conditions. Their eventual states, see Fig. 7, are then colored by red and blue, respectively. For $1<\bar{r}_{0}<1.132$, the epidemic occurs once every three years regardless of the initial conditions. As $\bar{r}_{0}$ races pass $1.132,(3(0,0,+), \mathrm{C})$-stability can be observed. For $1.317<$ $\bar{r}_{0}<\bar{r}_{+, 3} \approx 1.4151,(3(0,0,+), 8)$-stability can be noticed. For $\bar{r}_{+, 3}<\bar{r}_{0}<1.546$, 


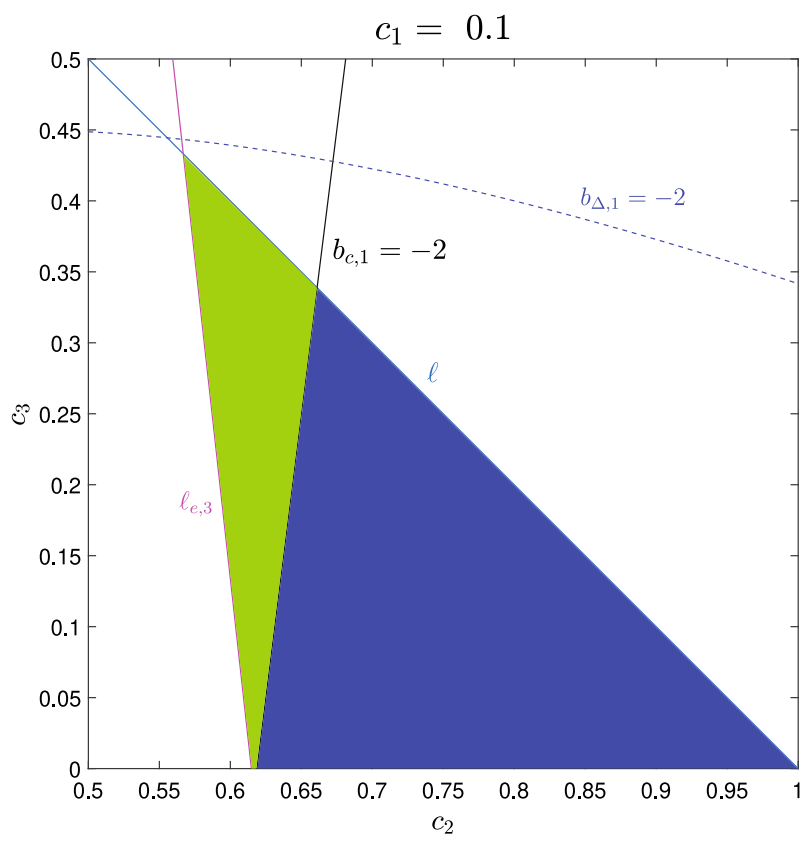

Fig. 4 Green region represents the $(1,3(0,0,+))$ stability where its boundaries are the lines $\ell_{e, 3}, b_{c, 1}=-2$ and $\ell$. In blue region, the period three points are stable. Moreover, it is where $(3(0,0,+), C)$ stability may occur. Here $0<c_{1}<2-\sqrt{3}$ (colour figure online)

as predicted, such period three points no longer exist. However, the chaotic dynamics seems to reappear with some windows occurring in between. As $\bar{r}_{0}$ races pass 1.546, the epidemic occurs every year with the size of infected population increasing as $\bar{r}_{0}$ becomes larger. Figure 8 provides the diagram of the maximum Lyapunov exponent of the map $\mathbf{F}$ versus $\bar{r}_{0}$ for the same set of parameters $c_{i}, i=1,2,3$ chosen in Fig. 7 . It then easy to see, via Figs. 7 and 8 , that $\left(3(0,0,+)\right.$, C)-stability occurs for $1.132<\bar{r}_{0}<1.317$ with $c_{i}, i=1,2,3$, fixed as above. Their correspondingly eventual states $\left(x_{n}, y_{n}\right)$, $1.95 \times 10^{4} \leq n \leq 2 \times 10^{4}$, with two sets of initial states are displayed in Fig. 9. We next fix $c_{1}=0.1$ and $\left(c_{2}, c_{3}\right)=(0.99,0.01)$, which is also from the blue region in Fig. 4. As a matter of fact, we see, via Fig. 10, that the chaotic dynamics appears only when period three points of $3(0,0,+)$ type no longer exist, i.e., $\bar{r}_{0}>\bar{r}_{+, 3}$. Hence, as compared to Fig. 7 , the existence of the $(3(0,0,+), C)$ stability is no longer there. In fact, chaotic dynamics can only be observed whenever $\bar{r}_{+, 3}<\bar{r}_{0}<1.843$. A period three points of $3(0,+,+)$ type, epidemic occurring twice every three years, emerges as $1.843<\bar{r}_{0}<2.185$. Whenever $2.185<\bar{r}_{0}<2.579$, the coexistence of the stable fixed point and this new type of period three points, i.e., $(1,3(0,+,+))$-stability, can be seen. Finally, as $\bar{r}_{0}$ becomes sufficiently large, the epidemic returns every year. In fact, Figs. 7 and 10 display qualitatively different bifurcation diagrams even though the parameters chosen are from the same region. 


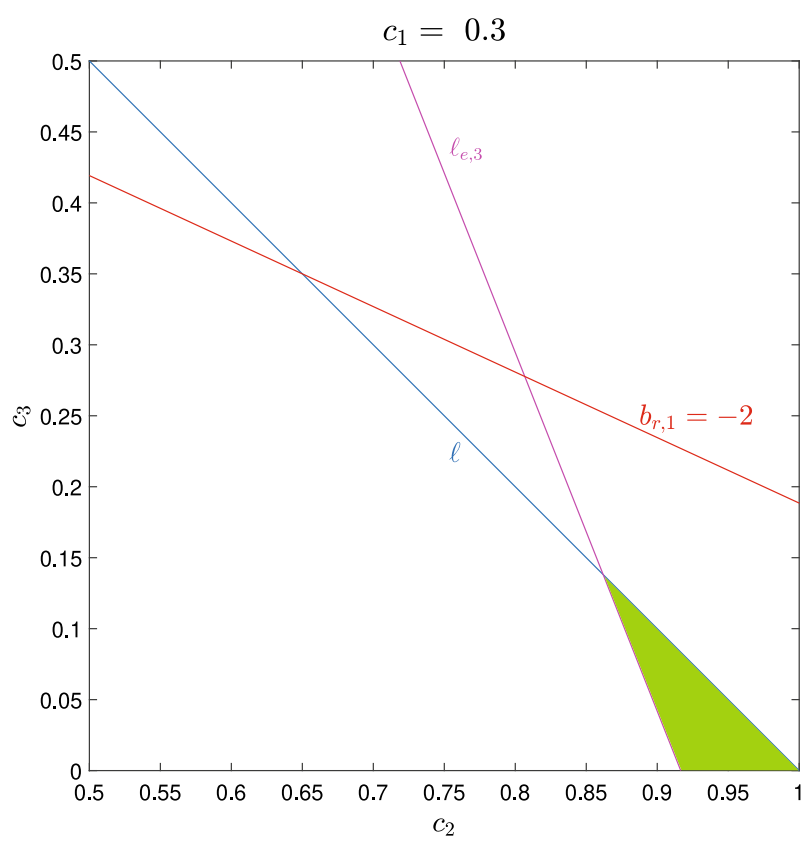

Fig. 5 Green region represents the $(1,3(0,0,+))$ stability where its boundaries are the lines $\ell_{e, 3}, \ell$ and the $c_{2}$-axis. Here $2-\sqrt{3} \leq c_{1} \leq c_{s_{1}, 3} \approx 3.19499$. In this range of parameters, existence of period three points implies $(1,3(0,0,+))$ stability (colour figure online)

\section{Conclusions}

In this paper, we analyze how the effect of parameters $\bar{r}_{0}$ and $c_{i}, i=1,2,3$, of the seasonal influenza map $\mathbf{F}$ has on its dynamics. If the adjusted basic reproduction number $\bar{r}_{0}$ is small ( $\left.\bar{r}_{0} \leq 1\right)$, the epidemic dies out every season. On the other hand, if the adjusted basic reproduction number $\bar{r}_{0}$ is sufficiently large, the epidemic returns every season. The most complicated and surprising dynamics occur when $\bar{r}_{0}$ is an intermediate value and the large portion of the populations in the same compartment changes in states the following season. Specifically, if more population in $P$ compartment move to the quickly recovered population $R^{\phi}(x)$ the following season than to these in the slowly recovered population $R^{1}(y)$, or equivalently $c_{2} \gg c_{3}$ and if the small portion of population in $R^{\phi}$ and $R^{1}$ compartments remain in the same compartments the following season, or equivalently $c_{1}, c_{2} \ll 1$, then the occurrence of the complicated dynamics can be observed whenever $\bar{r}_{0}$ is an intermediate value (see Fig. 8). In summary, our theoretical results and numerical simulations seem to suggest that heterogeneity of the population drives the complication and unpredictability of the dynamics.

We conclude this paper by mentioning some possible future work.

1. It is worthwhile to provide the bistability analysis of the following types: the existence of $(3(0,0,+), \mathrm{C})$-stability and $(1,3(0,+,+))$-stability. 


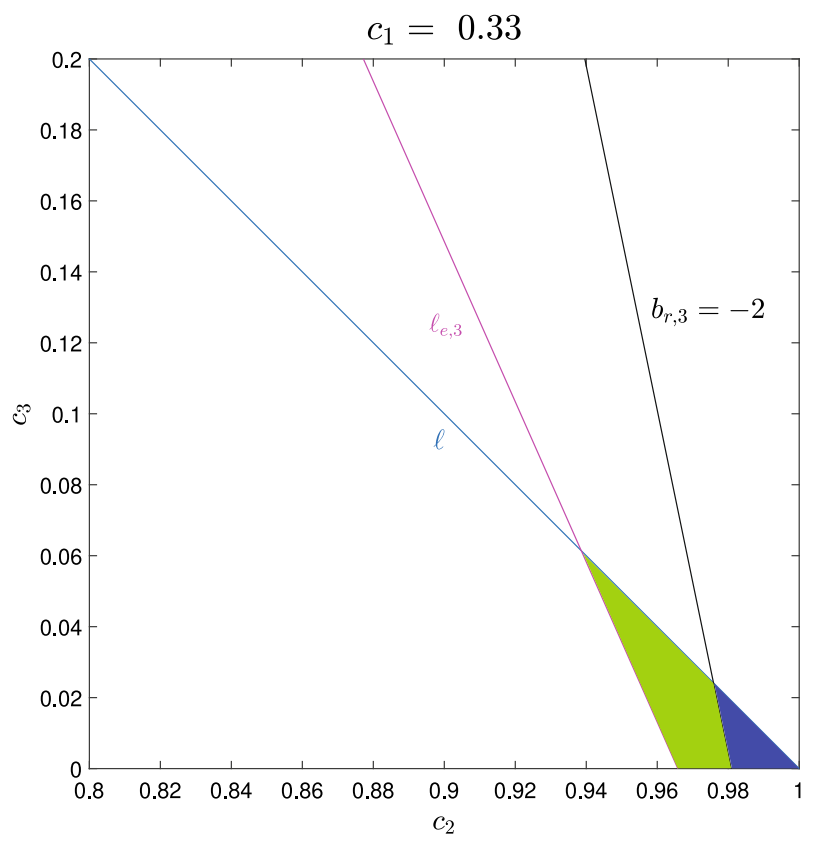

Fig. 6 Green region represents the $(1,3(0,0,+))$ stability where its boundaries are the lines $\ell_{e, 3}, \ell, b_{r, 3}=-2$ and the $c_{2}$-axis. Here $c_{s_{1}, 3}<c_{1}<c_{e, 3} \approx 0.35092$. In blue region, the fixed point is also stable (colour figure online)

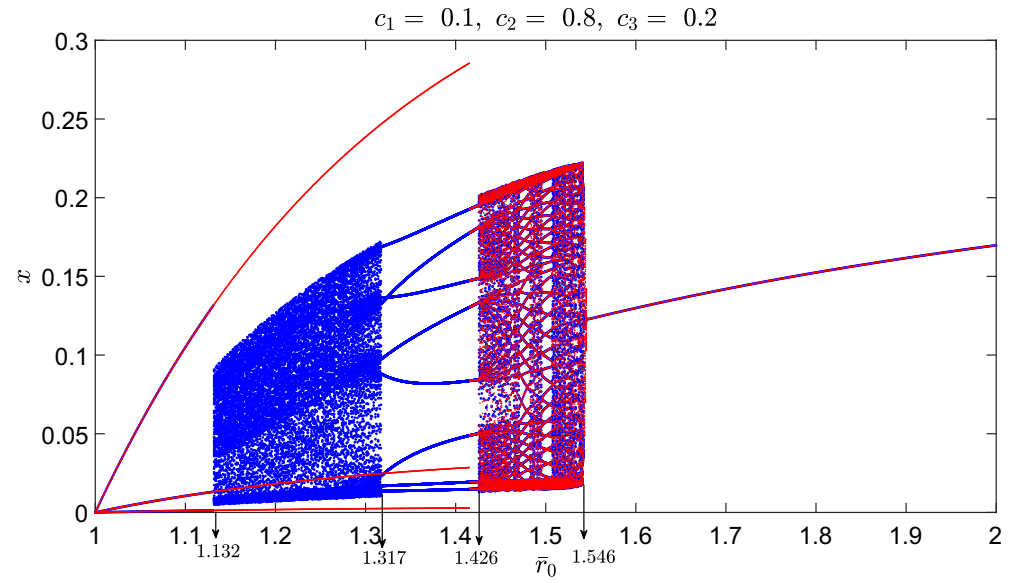

Fig. 7 Bifurcation diagram of the state of $x$ variable versus $\bar{r}_{0}$. Here the parameters $c_{1}=0.1, c_{2}=0.8$ and $c_{3}=0.2$, which are chosen from the blue region as given in Fig. 4 (colour figure online) 


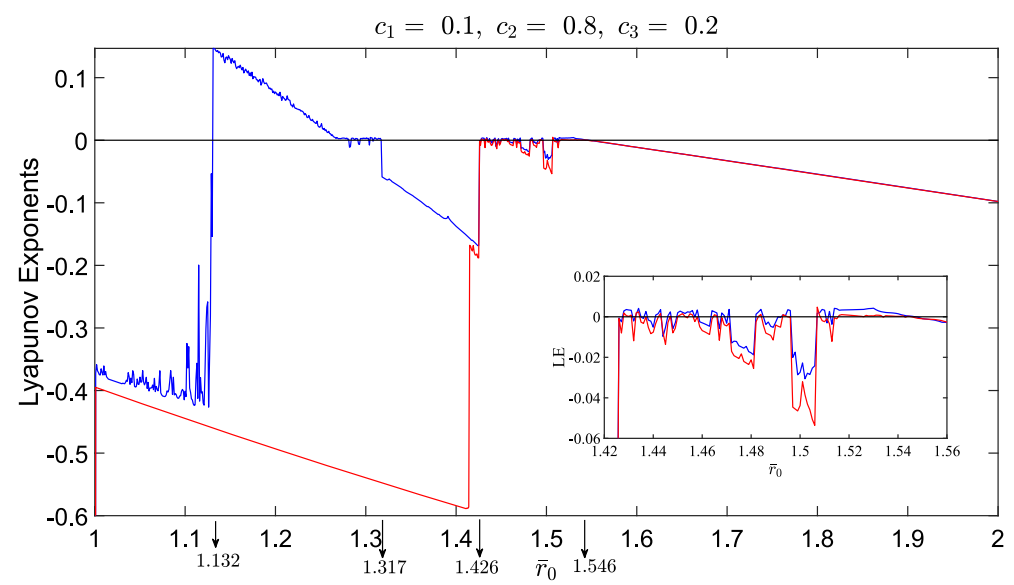

Fig. 8 Diagram of the maximum Lyapunov exponent (LE) of the map $\mathbf{F}$ versus $\bar{r}_{0}$. Here the parameters are chosen to be as those in Fig. 7

Fig. 9 Let $\left(x_{0}, y_{0}\right)=(0,0)$ and $\left(x_{0}, y_{0}\right)=(0.05,0.05)$, respectively. The eventual states of $\left(x_{n}, y_{n}\right), 1.95 \times 10^{4} \leq n \leq$ $2 \times 10^{4}$ and colored by red and blue, respectively, are displayed in the $x-y$ plane for the parameters with $c_{1}=0.1$, $c_{2}=0.8, c_{3}=0.2$, and $\bar{r}_{0}=1.2$ (colour figure online)

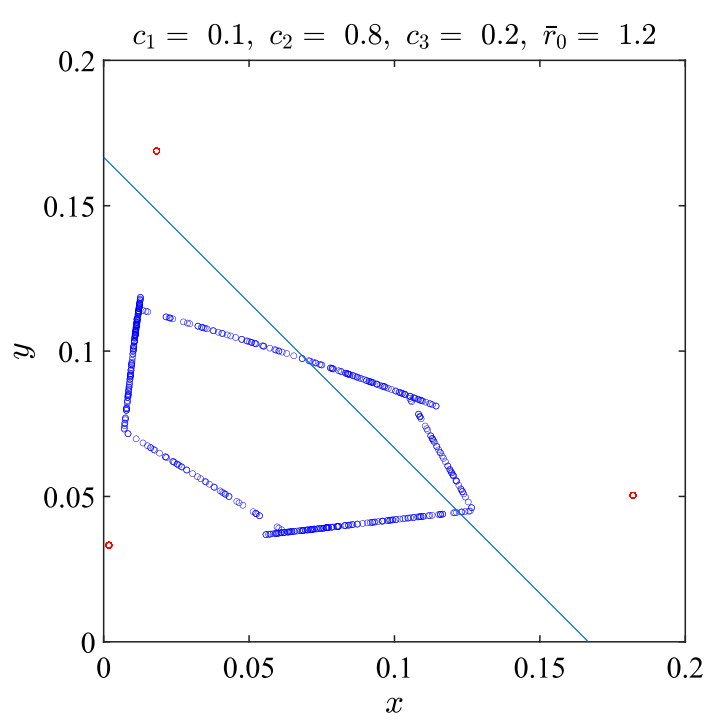

2. It is also interesting to investigate the global dynamics of the map $\mathbf{F}$. The first step toward this direction is to find the conditions for which the fixed point is globally stable.

3. The study of the maps defined in (17a)-(17d) or those obtained in (Roberts et al. 2019), which are algebraic-difference equations, is also valuable to the field of dynamics systems.

4. The undocumented infected individuals ( $\mathrm{Li}$ et al. 2020b) play a crucial role to facilitate the rapid dissemination of covid-19. Moreover, in real life or in the case of covid-19, some people infect many others and others do not spread the disease at all. That is why in addition to the basic reproduction number, a concept, named 


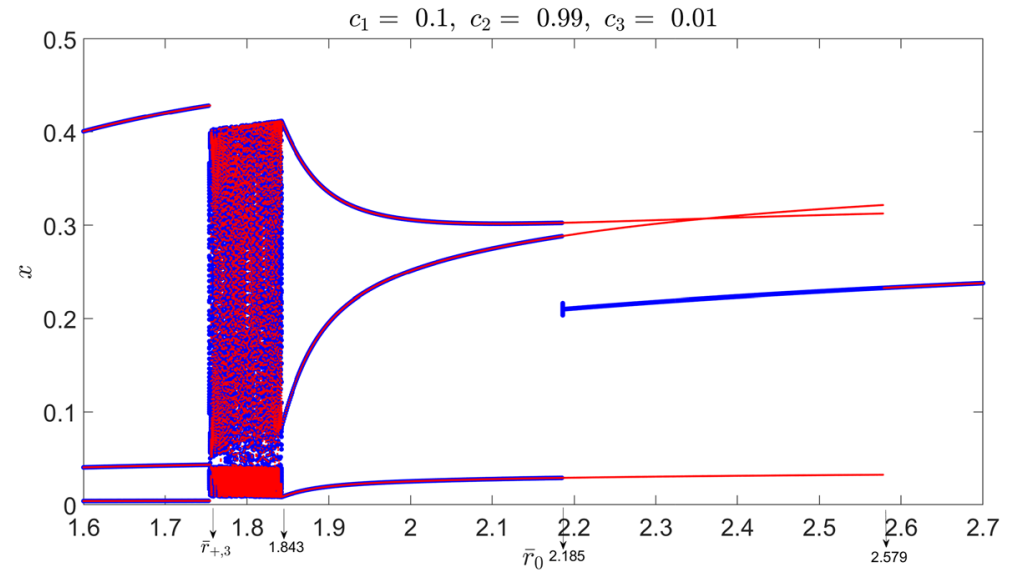

Fig. 10 Bifurcation diagram of the state of $x$ variable versus $\bar{r}_{0}$. Here the parameters $c_{1}=0.1, c_{2}=0.99$ and $c_{3}=0.01$ are also chosen from the blue region as given in Fig. 4. However, its dynamic is quite different from those in Fig. 7

dispersion number (Lloyd-Smith et al. 2005), of how much a disease clusters has to be factored in. In fact, the lower the dispersion number is, the more the transmission comes from a small number of people. It is of great interest to incorporate those ideas into the seasonal influenza models.

Funding Funding was provided by Ministry of Science and Technology, Taiwan (Grant No. MOST 1102115-M-A49-013).

\section{Appendix}

The following derivation of the map follows closely to the work done in Roberts et al. (2019). To obtain our seasonal model, we begin with the derivation of the relationship between the concerned population groups within a season. Let the proportion of the population that is susceptible to infection at time $t$ be $S(t)$ and the infected proportion be $I(t)$, to explore the effect of recovering from symptoms at different rates on an influenza epidemic as well as the effect of waning immunity. Such different rates of recovering may be due to the facts that people just recover from the flu differently or use non-drug approaches or even take wrong drug unintentionally or get two cooperative diseases, such as HIV virus and flu, in an overlapping period. We then further divide the proportion of the recovered population into two compartments, $R^{\phi}(t)$ and $R^{1}(t)$ with the implication that the people in the $R^{\phi}(t)$ compartment recover from the disease relatively quicker than those in $R^{1}(t)$ compartment. The population size is assumed to remain a constant, and so, $S(t)+I(t)+R^{\phi}(t)+R^{1}(t)=1$ for all $t>0$. The motion of the dynamics then reads as follows. 


$$
\begin{aligned}
\frac{\mathrm{d} S}{\mathrm{~d} t} & =-\beta S I, \\
\frac{\mathrm{d} I}{\mathrm{~d} t} & =\beta S I-\gamma I-k \gamma I, \\
\frac{\mathrm{d} R^{\phi}}{\mathrm{d} t} & =\gamma I, \\
\frac{\mathrm{d} R^{1}}{\mathrm{~d} t} & =k \gamma I .
\end{aligned}
$$

Here $k<1$ is the relative recovering factor for those in the $R^{1}$ compartment compared to those in the $R^{\phi}$. Note that if $k=0$, (14) reduces to the standard SIR model (see, e.g., Diekmann et al. 2013). The parameters $\beta$ and $\gamma$ are the transmission and recovering rates, respectively, for the disease. The basic reproduction number with respect to the standard SIR model is defined by $r_{0}=\frac{\beta}{\gamma}$. The outbreak of the epidemic takes off provided that $r_{0} S(0)>1+k$. Let $\lim _{t \rightarrow \infty}\left(S(t), R^{\phi}(t), R^{1}(t)\right)=$ $\left(S(\infty), R^{\phi}(\infty), R^{1}(\infty)\right)$. Note that it is easy to prove that $\lim _{t \rightarrow \infty} I(t)=0$ and the limits of $S(t), R^{\phi}(t)$, and $R^{1}(t)$ exist as $t$ goes to infinity. Then, it follows from the first and third equations in (14) that $S(t)=S(0) e^{-r_{0}\left(R^{\phi}(t)-R^{\phi}(0)\right)}$ and so

$$
R^{\phi}(\infty)=\frac{-1}{r_{0}} \ln \left(\frac{S(\infty)}{S(0)}\right)+R^{\phi}(0) .
$$

Similarly, we get

$$
R^{1}(\infty)=\frac{-k}{r_{0}} \ln \left(\frac{S(\infty)}{S(0)}\right)+R^{1}(0)
$$

To obtain our seasonal model, the final states of $R^{1}$ and $R^{\phi}$ the current season are to be used as the initial conditions for the following season. Let $P$ be the infected population at the end of the season. Then,

$$
\begin{aligned}
P & =S(0)-S(\infty)=\left(R^{\phi}(\infty)+R^{1}(\infty)\right)-\left(R^{\phi}(0)+R^{1}(0)\right) \\
& =\frac{-(1+k)}{r_{0}} \ln \left(\frac{S(\infty)}{S(0)}\right)=\frac{-(1+k)}{r_{0}} \ln \left(1-\frac{P}{S(0)}\right) .
\end{aligned}
$$

Hence,

$$
P=S(0)\left(1-e^{-r_{0} \frac{P}{1+k}}\right)=\left(1-R^{\phi}(0)-R^{1}(0)\right)\left(1-e^{-r_{0} \frac{P}{1+k}}\right)
$$

Note that $P=0$ is a trivial solution to Eq. (16). Moreover, if $\left(1-R^{\phi}(0)-\right.$ $\left.R^{1}(0)\right)\left(\frac{r_{0}}{1+k}\right)>1$ (resp., $<1$ ), then Eq. (16) has a unique positive (resp., negative) solution. 
The season-to-season map is to be constructed in terms of two recovered classes $R^{\phi}$ and $R^{1}$ with the assumption that those in $R^{\phi}$ compartment have the stronger immunity than those in $R^{1}$ compartment. Specifically, we assume that $c_{0}$ and $1-c_{0}$ portions of those in $R^{\phi}$ compartment at the beginning of the previous season remain in $R^{\phi}$ compartment and move to $R^{1}$ compartment, respectively, at the beginning of the following season. Here $0 \leq c_{0}<1$. Furthermore, $c_{1}$ and $1-c_{1}$ portions of those in $R^{1}$ compartment at the beginning of the previous season remain in $R^{1}$ compartment and move to $S$ compartment, respectively, at the beginning of the following season. Here $0 \leq c_{1}<1$. Finally, $c_{2}, c_{3}$, and $1-c_{2}-c_{3}$ portions of those in $P$ compartment at the end of the previous season move to $R^{\phi}, R^{1}$, and $S$ at the beginning of the following year, respectively. Here $0 \leq c_{2}, c_{3}<1$ and $c_{2}+c_{3} \leq 1$. Moreover, denote by $R_{n+1}^{\phi}$ and $R_{n+1}^{1}$ the size of $R^{\phi}$ and $R^{1}$, respectively, at time $n+1$. We then arrive at the following equations.

$$
\begin{aligned}
R_{n+1}^{\phi} & =c_{0} R_{n}^{\phi}+c_{2} P_{n}, \\
R_{n+1}^{1} & =\left(1-c_{0}\right) R_{n}^{\phi}+c_{1} R_{n}^{1}+c_{3} P_{n}, \\
P_{n} & = \begin{cases}\left(1-R_{n}^{\phi}-R_{n}^{1}\right)\left(1-e^{-\bar{r}_{0} P_{n}}\right), & \text { if }\left(1-R_{n}^{\phi}-R_{n}^{1}\right) \bar{r}_{0}>1, \\
0, & \text { otherwise. }\end{cases}
\end{aligned}
$$

Here

$$
\bar{r}_{0}=\frac{r_{0}}{1+k} .
$$

The newly defined term $\bar{r}_{0}$ is to be called the adjusted basic reproduction number.

Clearly, (17a), (17b) and (17c) define an algebraic-difference equation modeling the dynamics of a between-season influenza.

We next seek to find an approximate solution $\tilde{P}_{n}$ to equation $(17 \mathrm{c})$. To this end, we replace $e^{-\bar{r}_{0} P_{n}}$ in (17c) by its Taylor polynomial $1-\bar{r}_{0} P_{n}+\frac{\bar{r}_{0}^{2}}{2} P_{n}^{2}$ of degree 2 . Then, the approximate equation to equation $(17 \mathrm{c})$ reduces to $\left(1-R_{n}^{\phi}-R_{n}^{1}\right)\left(\bar{r}_{0}-\frac{\bar{r}_{0}^{2}}{2} P_{n}\right)=1$. Denote the solution to the above approximate equation by $\tilde{P}_{n}$. We then get the following approximate model.

$$
\begin{aligned}
& R_{n+1}^{\phi}=c_{0} R_{n}^{\phi}+c_{2} \tilde{P}_{n}, \\
& R_{n+1}^{1}=\left(1-c_{0}\right) R_{n}^{\phi}+c_{1} R_{n}^{1}+c_{3} \tilde{P}_{n}, \\
& \tilde{P}_{n}= \begin{cases}\frac{2}{\bar{r}_{0}}\left(1-\frac{1}{\bar{r}_{0}\left(1-R_{n}^{\phi}-R_{n}^{1}\right)}\right), & \text { if } R_{n}^{\phi}+R_{n}^{1} \leq 1-\frac{1}{\bar{r}_{0}}, \\
0, & R_{n}^{\phi}, R_{n}^{1} \geq 0, \\
\text { if } 1-\frac{1}{\bar{r}_{0}} \leq R_{n}^{\phi}+R_{n}^{1} \leq 1, \\
R_{n}^{\phi}, R_{n}^{1} \geq 0 .\end{cases}
\end{aligned}
$$


To save notations, we shall denote $R^{\phi}, R^{1}$, and $\tilde{P}$ by $x, y$, and $p$, respectively. Writing (18) into a compact form with the new notations, we then arrive at the iterative map defined in (1a)-(1c).

The reasons to use such approximation are twofold. First, each flu season lasts only finite time. However, (17c) or (16) is derived from within-season model (14) as time approaches to infinity. It should also be noted that the size of the infected population is increasing in time. Therefore, in real situation, the size of the infected population at the end of each season should be smaller than the one produced from (16). It can be easily verified that $\tilde{P}_{n}$, as defined in (18c), is smaller than those obtained from (17c). Hence, it is more feasible to consider approximate model (1). Second, it is obvious that the map defined in (1) is easier to analyze analytically than that of defined in (17). Nevertheless, to analyze map $\mathbf{F}$ is still a nontrivial matter, because that the map F consists of both linear and nonlinear dynamics and which one of the types is to be acted on at time $n+1$ depends upon the position of the previous iterate $\left(x_{n}, y_{n}\right)$. Specifically, if $x_{n}+y_{n} \leq 1-\frac{1}{\bar{r}_{0}}$, then its corresponding the dynamics is nonlinear.

Otherwise, the map $\mathbf{F}$ reduces to the linear map $\mathbf{C}$, as given in (1b).

\section{References}

Agarwal, R.P.: Dynamical Systems and Applications. World Scientific, Singapore (1995)

Anzo-Hernández, A., Gilardi-Velázquez, H.E., Campos-Cantón, E.: On multistability behavior of unstable dissipative systems. Chaos (2018). https://doi.org/10.1063/1.5016329

Bao, H., Hua, Z., Wang, N., Zhu, L., Chen, M., Bao, B.: Initials-boosted coexisting Chaos in a 2-D sine map and its hardware implementation. IEEE Trans. Ind. Inform. 17, 1132-1140 (2021). https://doi. org/10.1109/TII.2020.2992438

Casas, G.A., Rech, P.C.: Multistability annihilation in the Hénon map through parameters modulation. Commun. Nonlinear Sci. Numer. Simul. 17, 2570-2578 (2012). https://doi.org/10.1016/j.cnsns.2011. 10.031

Diekmann, O., Heesterbeek, H., Britton, T.: Mathematical Tools for Understanding Infectious Disease Dynamics. Princeton University Press, Princeton (2013)

Escalante-González, R.J., Campos, E.: Multistable systems with hidden and self-excited scroll attractors generated via piecewise linear systems. Complexity (2020). https://doi.org/10.1155/2020/7832489

Gilardi-Velázquez, H.E., Ontañón-García, L.J., Hurtado-Rodriguez, D.G., Campos-Cantón, E.: Multistability in piecewise linear systems versus eigenspectra variation and round function. Int. J. Bifurc. Chaos 27, 1730031 (2017). https://doi.org/10.1142/S0218127417300312

Jackson, T., Radunskaya, A.: Applications of Dynamical Systems in Biology and Medicine. Springer, Berlin (2015)

Li, H., Hua, Z., Bao, H., Zhu, L., Chen, M., Bao, B.: Two-dimensional memristive hyperchaotic maps and application in secure communication. IEEE Trans. Ind. Electron. (2020a). https://doi.org/10.1109/ TIE.2020.3022539

Li, R., Pei, S., Chen, B., Song, Y., Zhang, T., Yang, W., Shaman, J.: Substantial undocumented infection facilitates the rapid dissemination of novel coronavirus (SARS-CoV-2). Science 368, 489-493 (2020b). https://doi.org/10.1126/science.abb3221

Lloyd-Smith, J.O., Schreiber, S.J., Kopp, P.E., Getz, W.M.: Superspreading and the effect of individual variation on disease emergence. Nature 438, 355-359 (2005). https://doi.org/10.1038/nature04153

Martins, L.C., Gallas, J.A.C.: Multistability phase diagrams and statistical properties of the kicked rotor: a map with many coexisting attractors. Int. J. Bifurc. Chaos 18, 1705-1717 (2008). https://doi.org/10. 1142/S0218127408021294

Mattioni, F., Weaver, P.M., Potter, K.D., Friswell, M.I.: The application of thermally induced multistable composites to morphing aircraft structures. Proc. SPIE (2008). https://doi.org/10.1117/12.776226 
Morfu, S., Nofiele, B., Marquié, P.: On the use of multistability for image processing. Phys. Lett. A 367, 192-198 (2007). https://doi.org/10.1016/j.physleta.2007.02.086

Natiq, H., Said, M.R.M., Ariffin, M.R.K., He, S., Rondoni, L., Banerjee, S.: Self-excited and hidden attractors in a novel chaotic system with complicated multistability. Eur. Phys. J. Plus 133, 557 (2018). https://doi.org/10.1140/epjp/i2018-12360-y

Natiq, H., Banerjee, S., Ariffin, M.R.K., Said, M.R.M.: Can hyperchaotic maps with high complexity produce multistability? Chaos (2019). https://doi.org/10.1063/1.5079886

Rahim, M.F.A., Natiq, H., Fataf, N.A.A., Banerjee, S.: Dynamics of a new hyperchaotic system and multistability. Eur. Phys. J. Plus 134, 499 (2019). https://doi.org/10.1140/epjp/i2019-13005-5

Roberts, M.G., Hickson, R.I., McCaw, J.M., Talarmain, L.: A simple influenza model with complicated dynamics. J. Math. Biol. 78, 607-624 (2019). https://doi.org/10.1007/s00285-018-1285-z

Santos-Moreno, J., Tasiudi, E., Stelling, J., Schaerli, Y.: Multistable and dynamic CRISPRi-based synthetic circuits. Nat. Commun. 11, 2746 (2020). https://doi.org/10.1038/s41467-020-16574- 1

Strogatz, S.H.: Nonlinear Dynamics and Chaos: with Applications to Physics, Biology, Chemistry, and Engineering (Studies in Nonlinearity). Westview Press, Boulder (2001)

Swishchuk, A., Islam, S.: Random Dynamical Systems in Finance. Chapman and Hall, London (2013)

Weisshaar, T.A.: Morphing aircraft systems: historical perspectives and future challenges. J. Aircr. 50, 337-353 (2013). https://doi.org/10.2514/1.C031456

Zhang, Y., Luo, G.: Multistability of a three-degree-of-freedom vibro-impact system. Commun. Nonlinear Sci. Numer. Simul. 57, 331-341 (2018). https://doi.org/10.1016/j.cnsns.2017.10.007

Publisher's Note Springer Nature remains neutral with regard to jurisdictional claims in published maps and institutional affiliations. 